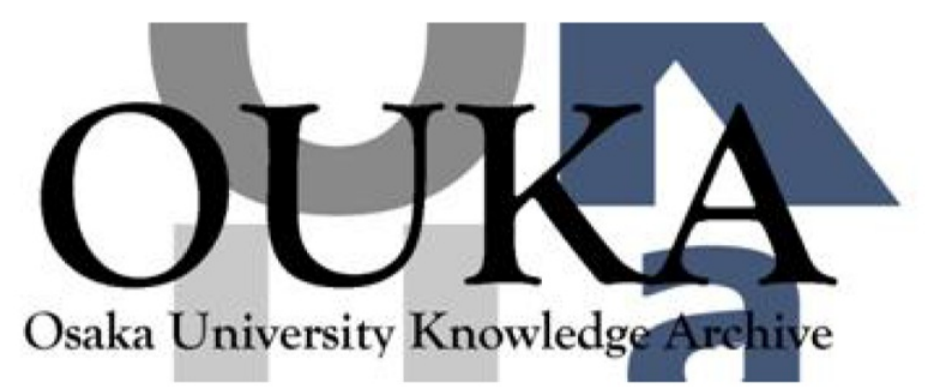

\begin{tabular}{|c|l|}
\hline Title & $\begin{array}{l}\text { Ion distribution function in a weakly } \\
\text { collisional sheath }\end{array}$ \\
\hline Author(s) & Hamaguchi, S.; Farouki, R. T.; Dalvie, M. \\
\hline Citation & Physical Review A. 44(6) p. 3804-p. 3821 \\
\hline Issue Date & $1991-09$ \\
\hline oaire:version VoR \\
\hline URL & https://hdl. handle.net/11094/78522 \\
\hline rights & $\begin{array}{l}\text { Copyright (1991) by the American Physical } \\
\text { Society }\end{array}$ \\
\hline Note & \\
\hline
\end{tabular}

Osaka University Knowledge Archive : OUKA

https://ir. Library. osaka-u. ac. jp/

Osaka University 


\title{
Ion distribution function in a weakly collisional sheath
}

\author{
S. Hamaguchi, R. T. Farouki, and M. Dalvie \\ IBM Thomas J. Watson Research Center, Yorktown Heights, New York 10598
}

(Received 11 March 1991)

\begin{abstract}
The ion distribution function in a weakly collisional sheath is obtained analytically for arbitrary electric-field configurations, based on kinetic equations with elastic collisions. In the light of application to plasma etching and deposition, the distribution function is integrated to derive the angular and energy distributions of the ion flux incident at the electrodes. Under the assumption of a constant-sheath electric field, the simple analytic expressions $\Gamma_{\theta} \propto 2 \sin \theta \cos \theta\left(-4 \ln \sin \theta+\sin ^{4} \theta-1\right) /\left(1+\sin ^{2} \theta\right)^{2}$ and $\Gamma_{\mathrm{en}} \propto-\ln (1-\eta)$ for the angular and energy distributions of the nonballistic ion flux are obtained, where $\eta$ denotes the normalized ion kinetic energy. For the more realistic case of a self-consistent electric field, integral formulas for $\Gamma_{\theta}$ and $\Gamma_{\mathrm{en}}$, which are amenable to numerical quadrature, are also obtained from the Boltzmann-Poisson system. These analytic expressions are compared with Monte Carlo sheath simulations under various conditions and are found to be in excellent agreement.
\end{abstract}

\section{INTRODUCTION}

Low-temperature, partially ionized plasmas are widely used in the fabrication of VLSI (very-large-scaleintegration) circuits. As electronic technology advances and the sizes of integrated circuits diminish, anisotropy in plasma processing (i.e., unidirectionality of the cathode-bombarding ions) becomes an increasingly important requirement. In recent years, high-density, weakly collisional plasmas in state-of-the-art process tools, including ECR (electron cyclotron resonance) devices, have shown promise in improving anisotropy over conventional rf (radio frequency) plasma tools. In such fabrication tools, the outcome of the process depends strongly on the nature of the plasma sheath located between the bulk plasma and the processed material. A quantitative understanding of plasma sheaths is thus of practical importance in controlling fabrication processes.

In high-density low-pressure processing tools, most ions impinging on the electrodes are unidirectional or ballistic, being accelerated by the sheath electric field. However, the ions that do collide with neutrals give rise to an off-normal component that can influence the shape of submicrometer features on processed materials. Such collided ions in weakly collisional sheaths are evidently far from thermalized and thus far from Maxwellian, so their distribution may be obtained theoretically only through solving the kinetic equation describing the system.

To date, much effort has been made to measure and compute the ion distribution functions in sheaths of various glow discharges. Davis and Vanderslice [1] made the first systematic measurements of the energy distribution of ions striking the cathode and found that the energy distributions vary considerably according to the collisionality (i.e., the ratio of the sheath thickness to the mean free path). Taking into account only chargeexchange collisions, Davis and Vanderslice also proposed a simple model of the ion distribution and succeeded in explaining some of the measured energy distributions.

Taking a more microscopic view, Kushner [2] computed the energy and angular distributions of the impinging ions in $\mathrm{rf}$ discharges, using the Monte Carlo technique. In his calculations, as in the model of Davis and Vanderslice, only charge-exchange collisions are taken into account, which only yield small angular scattering due to the neutral thermal energy.

More comprehensive Monte Carlo simulations were presented by Thompson, Sawin and Fisher [3], which included several different forms of elastic ion-neutralspecies interactions as well as charge-exchange collisions in $\mathrm{dc}$ and $\mathrm{rf}$ discharges with predefined electric fields. Recent measurements of the angular distribution in $\mathrm{rf}$ discharges by Liu, Huppert, and Sawin [4] have indicated that some discrepancy exists between the measured distribution and the angular distribution computed by the Monte Carlo method similar to that of Thompson, Sawin, and Fisher [3]. Farouki, Hamaguchi, and Dalvie [5] have recently reported Monte Carlo simulations with selfconsistent electric fields due to space-charge effects, taking into account both elastic scattering and chargeexchange collisions, and discussed the Monte Carlo results in the context of kinetic theory.

In contrast to such considerable efforts in numerical simulation and experimental measurement of the ion distributions in sheaths, it appears that analytic treatment of the sheath ion kinetics has been slow in progress. This is mainly due to the difficulty in calculating the collision integral of the Boltzmann equation describing ionneutral-species collisions, which generally give rise to strong anisotropy of ion distribution in the velocity space. As Druyvesteyn and Penning and several other authors [6]-[10] have shown, calculation of the distribution functions involving only colliding particles with a small mass ratio, such as the electron-neutral-species scattering, is amenable to expansion about an isotropic distribution. However, when the mass ratio of two colliding particles is not ignorable, which is the case for most 
ion-neutral-species collisions, such expansion methods generally fail. Wannier [11] solved the Boltzmann equation for ions with ion-neutral-species elastic collisions in the limit of a strong uniform electric field. Lawler [12] also solved the Boltzmann equation with chargeexchange collisions and obtained an ion distribution function whose asymptotic limit is Wannier's equilibrium solution.

In the present work, we solve the Boltzmann equation with elastic collisions to obtain the analytic expression of the ion distribution function in a weakly collisional sheath. Under the assumption of low collisionality, the Boltzmann equation is expanded in terms of the small parameter $d / \lambda_{\text {MFP }}$ (i.e., the ratio of the sheath thickness to the ion mean free path). Assuming that the lowest-order solution of the Boltzmann equation be the ballistic ions passing through the sheath as a beam, we are able to derive the distribution function of the scattered ions in an explicit form for arbitrary electric-field configurations, including the self-consistent electric field. In high-density low-pressure processing plasmas, the collisionality parameter $d / \lambda_{\mathrm{MFP}}$ is typically $10^{-1}-10^{-3}$. For example, when the neutral density is $n_{g}=2.7 \times 10^{13} \mathrm{~cm}^{-3}$ at a gas pressure $p_{g}=1 \mathrm{mTorr}$ and temperature $T_{g}=350 \mathrm{~K}$, the total cross section for elastic collisions in Ar discharges $\sigma_{\text {tot }} \simeq 5 \times 10^{-15} \mathrm{~cm}^{2}[13,14]$ gives a mean free path $\lambda_{\mathrm{MFP}}=\left(n_{g} \sigma_{\text {tot }}\right)^{-1}=73.5 \mathrm{~mm}$, and with a typical sheath thickness $d=0.1 \mathrm{~mm}$, we have $d / \lambda_{\mathrm{MFP}}=1.4 \times 10^{-3}$, which is typical in ECR discharges. The smallness of the parameter $d / \lambda_{\text {MFP }}$ required for the theory presented in this work to be valid is also discussed; it is shown that when $d / \lambda_{\mathrm{MFP}} \simeq 0.2$ or less, the analytic angular and energy distributions are in remarkably good agreement with the distributions obtained numerically from the Monte Carlo simulations, and up to $d / \lambda_{\mathrm{MFP}} \simeq 1$ the analytic expressions may still be used as good approximations.

The goal of this paper is to treat in a mathematically rigorous manner a model system that singles out the physics of plasma sheaths in which ion-neutral-species elastic scattering dominates. To achieve this goal, we need to make some simplifications and idealizations, disposing of such effects as one may treat elsewhere as separate problems or easily include in numerical codes as secondary effects. The first idealization that we make is to consider only hard-sphere elastic collisions in the sheath. It is generally considered that, for noble gases, the cross section for charge-exchange collisions is of the order of, or larger than, that of elastic collisions. However, we confine ourselves here to the problem of elastic collisions since large-angle scattering, which is the major factor in determining the anisotropy of processing plasmas, results mostly from elastic collisions. Furthermore, as shown in [5], the complete ion distribution function is a simple superposition of the distribution functions calculated separately with the elastic collisions and chargeexchange collisions. As stated earlier, the ion distribution functions with the charge-exchange collisions are calculated analytically in [12] and numerically in [1]-[3] and [5]. We also point out that it is reported in [3] that different types of elastic collisions, such as hard sphere and soft sphere, do not significantly alter the resulting ion distributions.

We also assume for the sake of brevity that the sheath is composed purely of ions, defining the presheath-sheath boundary as an interface beyond which electrons are significantly depleted. Technically, as will be shown later, it would not be a difficult task to include a simple model of electrons in our kinetic formulation, such as electrons in equilibrium with the electric field, in order to incorporate a more realistic model of the cathode fall region. Discussions on more complex electron distribution functions are also found, for example, in [15-17]. However, in view of our goal in this work, we postpone inclusion of realistic models for the presheath and other electron-related phenomena, such as ionization and dissociation, to future studies.

Although most processing plasmas are generated by rf discharges, we consider in this work only steady-state solutions of the kinetic equations, modeling dc discharges. However, in certain parameter regimes of $\mathrm{rf}$ discharges, the ion transit time can be significantly longer than or shorter than the rf period, such that ions experience essentially time-averaged or instantaneous electric fields of the rf cycle. Therefore, except for some resonant phenomena that may occur when the ion transit time across the rf sheath is close to the rf period, dc sheath models still play an important role in understanding some basic rf discharge properties [18,19].

In the light of application to plasma etching and deposition, important quantities to calculate from the kinetic equations are the angular and energy distributions of the ion flux impinging on the electrodes on which processed materials are placed. It is also of interest to calculate the angular and energy distributions of the ion density, which are simple integrations over the magnitude of the velocity for the angular distribution, and over the angle for the energy distribution, of the ion distribution function $f$ which is the solution of the Boltzmann equation. In this paper we obtain, by solving the Boltzmann equation for weakly collisional sheaths, analytic formulas for the angular distribution $\Gamma_{\theta}(\theta)$ and energy distribution $\Gamma_{\mathrm{en}}(\eta)$ of the ion flux, namely

$$
\Gamma_{\theta}(\theta) \propto \frac{2 \sin \theta \cos \theta}{\left(1+\sin ^{2} \theta\right)^{2}}\left[4 \ln \frac{1}{\sin \theta}+\sin ^{4} \theta-1\right]
$$

and

$$
\Gamma_{\mathrm{en}}(\eta) \propto-\ln (1-\eta)
$$

where $\eta$ is the kinetic energy of an impinging ion normalized by its maximum value. Although obtained under the assumption of a constant electric field, these formulas are also a good approximation for more realistic selfconsistent fields. The general expressions of the ion distributions in self-consistent electric fields are also derived in this work. These expressions of the ion flux serve as an inexpensive means to predict the effects of the ion bombardment on electrode surfaces. For example, the distributions of the ion flux derived in this work may be used as input data for shape-evolution calculations, such as 
those described in [20].

The rest of this paper is organized as follows. In Sec. II, model equations for the sheath are presented based on kinetic theory. The ion distribution function is derived from these model equations in Sec. III. The angular and energy distributions are calculated by integrating the ion distribution function in Secs. IV and V, respectively, and these analytic formulas are compared to the Monte Carlo simulation results. Section VI contains the conclusions and discussion.

\section{KINETIC EQUATIONS}

As a model of a plasma sheath, we consider a steadystate $(\partial / \partial t=0)$, capacitively coupled planar discharge with the electric field in the $z$ direction and no magnetic field. The ion distribution function $f(\mathbf{v}, z)$ is then governed by the Boltzmann equation coupled with Poisson's equation:

$$
\begin{aligned}
& v_{z} \frac{\partial f}{\partial z}-\frac{q}{m} \frac{d \Phi}{d z} \frac{\partial f}{\partial v_{z}}=\left[\frac{\delta f}{\delta t}\right]_{\mathrm{col}}, \\
& \frac{d^{2} \Phi}{d z^{2}}=-\frac{q n(z)}{\epsilon_{0}} .
\end{aligned}
$$

Here the ion density $n(z)$ at $z$ is given by

$$
n(z)=\int f(\mathbf{v}, z) d \mathbf{v},
$$

and the collision integral $(\delta f / \delta t)_{\text {col }}$ may be expressed as

$$
\begin{aligned}
{\left[\frac{\delta f}{\delta t}\right]_{\mathrm{col}}=} & \int F\left(\mathbf{V}^{\prime}\right) f\left(\mathbf{v}^{\prime}\right)\left|\mathbf{v}^{\prime}-\mathbf{V}^{\prime}\right| \sigma d^{2} \Omega \frac{\partial\left(\mathbf{v}^{\prime}\right)}{\partial(\mathbf{v})} d \mathbf{V}^{\prime} \\
& -\int F(\mathbf{V}) f(\mathbf{v})|\mathbf{v}-\mathbf{V}| \sigma d^{2} \Omega d \mathbf{V}
\end{aligned}
$$

where the Jacobian has the form

$$
\frac{\partial\left(\mathbf{v}^{\prime}\right)}{\partial(\mathbf{v})}=\left[\frac{\left|\mathbf{v}^{\prime}-\mathbf{v}^{\prime}\right|}{\left|\mathbf{v}-\mathbf{v}^{\prime}\right|}\right]^{3}
$$

In Eq. (4), $\sigma$ denotes the differential cross section for ion-neutral-species collisions, $\Omega$ denotes scattering solid angle, $F$ denotes the distribution function of neutrals, $\mathbf{V}$ and $\mathbf{v}$ denote the velocities of neutrals and ions, respectively, and the primed quantities $\mathbf{v}^{\prime}$ and $\mathbf{V}^{\prime}$ indicate velocities before collisions. Here the ion-neutral-species collisions are assumed to be elastic. The $z$ dependence of the distribution functions is not explicitly indicated in Eq. (4). The first term on the right-hand side of Eq. (4) represents the rate of increase of ions in an infinitesimal velocityspace volume element $d \mathbf{v}$ due to scattering into that element by ion-neutral-species collisions, whereas the second term represents the rate of decrease of ions in $d \mathbf{v}$ due to scattering out of that element by ion-neutralspecies collisions. For further details on the collision integral, the reader may refer to, for example, [21].

It is easy to show that the integral of the collision term $(\delta f / \delta t)_{\text {col }}$ over the entire velocity space $\mathbf{v}$ vanishes. From Eq. (1), therefore, the $z$ component of the ion flux

$$
\Gamma_{0}=\int v_{z} f d \mathbf{v}=n(z)\left\langle v_{z}\right\rangle(z)
$$

is seen to be independent of $z$ and solely determined by the conditions at the presheath-sheath boundary, $z=0$. The right-hand side of Eq. (6) gives the definition of the ion stream velocity $\left\langle v_{z}\right\rangle(z)$.

In this paper, we consider only uniformly distributed (i.e., $z$-independent) cold neutrals, so that

$$
F(\mathbf{V})=n_{g} \delta(\mathbf{V}) \text {, }
$$

where $n_{g}$ denotes the neutral number density. Executing integration of Eq. (4) with $F$ given above, we obtain

$$
\left[\frac{\delta f}{\delta t}\right]_{\mathrm{col}}=n_{g} \sigma_{\mathrm{tot}} v\left[\int\left[\frac{v^{\prime}}{v}\right]^{4} f\left(\mathbf{v}^{\prime}\right) \frac{\sigma}{\sigma_{\mathrm{tot}}} d^{2} \Omega-f(\mathbf{v})\right],
$$

where $v=|\mathbf{v}|, v^{\prime}=\left|\mathbf{v}^{\prime}\right|$ and $\sigma_{\text {tot }}$ denotes the total cross section. In the case of hard-sphere collisions, the differential cross section of the ions scattered into the solid angle element $d^{2} \Omega$ may be written [22] as

$$
\sigma d^{2} \Omega=\frac{\sigma_{\text {tot }}}{4 \pi} \sin \chi_{\text {c. m. }} d \chi_{\text {c. } . .} d \psi
$$

where $\chi_{\text {c.m. }}$ is the polar scattering angle measured in the center-of-mass system and $\psi$ is the azimuthal scattering angle about the $\mathbf{v}^{\prime}$ direction. The total cross section $\sigma_{\text {tot }}$ is given in terms of the sum $a$ of the ion and neutral radii by $\sigma_{\text {tot }}=\pi a^{2}$. The center-of-mass scattering angle $\chi_{\text {c.m. }}$ is related to the ion velocities $v^{\prime}$ and $v$ by

$$
\frac{v}{v^{\prime}}=\frac{\sqrt{m^{2}+M^{2}+2 m M \cos \chi_{\text {c.m. }}}}{m+M},
$$

with $m$ and $M$ being the ion and neutral masses, respectively, and to the scattering angle in the laboratory frame $\chi$ by

$$
\tan \chi=\frac{M \sin \chi_{\text {c.m. }}}{m+M \cos \chi_{\text {c.m. }}}
$$

In the special case $m=M$ that we shall be primarily concerned with, we have

$$
\chi_{\text {c.m. }}=2 \chi(0 \leq \chi \leq \pi / 2) \text { and } v=v^{\prime} \cos \chi .
$$

The system of Eqs. (1)-(3) may be solved with the "initial" conditions

$$
f(\mathbf{v}, 0)=f_{I}(\mathbf{v}), \quad \Phi(0)=0, \quad \text { and } \quad \frac{d \Phi}{d z}=E_{I}
$$

given at $z=0$, where $E_{I}$ denotes the magnitude of the electric field at the presheath-sheath boundary.

We now nondimensionalize Eqs. (1) - (3) by using the following transformations:

$$
\begin{aligned}
& f=\bar{f} n_{I} /\left(\omega_{p i} d\right)^{3}, \quad n=\bar{n} n_{I}, \quad \Phi=\phi q n_{I} d^{2} / \epsilon_{0}, \\
& \mathbf{v}=\mathbf{u} \omega_{p i} d, \quad z=\zeta d,
\end{aligned}
$$

where $n_{I}$ is the ion density at the presheath-sheath boundary, $n_{I}=n(0), d$ is the sheath thickness, and $\omega_{p i}=\left(q^{2} n_{I} / \epsilon_{0} m\right)^{1 / 2}$ is the ion plasma frequency. Using the dimensionless variables in Eqs. (12), the system of 
Eqs. (1)-(3) may be written as

$$
\begin{aligned}
& u_{z} \frac{\partial \bar{f}}{\partial \zeta}-\frac{d \phi}{d \xi} \frac{\partial \bar{f}}{\partial u_{z}} \\
& =\frac{d}{\lambda_{\mathrm{MFP}}} u\left[\int\left[\frac{u^{\prime}}{u}\right]^{4} \bar{f}\left(\mathbf{u}^{\prime}\right) \frac{\sigma}{\sigma_{\mathrm{tot}}} d^{2} \Omega-\bar{f}(\mathbf{u})\right], \\
& \frac{d^{2} \phi}{d \xi^{2}}=-\bar{n}(\zeta) \text {, } \\
& \bar{n}(\zeta)=\int \bar{f}(\mathbf{u}, \zeta) d \mathbf{u},
\end{aligned}
$$

where $u=|\mathbf{u}|, \quad u^{\prime}=\left|\mathbf{u}^{\prime}\right|$, and $\lambda_{\mathrm{MFP}}=\left(n_{g} \sigma_{\text {tot }}\right)^{-1}$ is the mean free path. The initial conditions in Eq. (11) are also rescaled accordingly.

\section{THE ION DISTRIBUTION FUNCTION IN A WEAKLY COLLISIONAL SHEATH}

We now solve Eqs. (13)-(15) in the limit of weak collisionality, $\epsilon=d / \lambda_{\mathrm{MFP}}<<1$. Henceforth the ions and the neutrals are assumed to be of the same species, so that $m=M$. For the sake of simplicity, the ions are assumed to enter the sheath with a fixed velocity $v_{B}$ as a beam, so the "initial" condition for $f$ is given by

$$
f_{I}(\mathbf{v})=\frac{n_{I} \delta\left(v_{\perp}\right)}{2 \pi v_{\perp}} \delta\left(v_{z}-v_{B}\right)
$$

or

$$
\bar{f}(\mathbf{u}, \zeta=0)=\frac{\delta\left(u_{\perp}\right)}{2 \pi u_{\perp}} \delta\left(u_{z}-u_{B}\right),
$$

with $u_{B}=v_{B} / \omega_{p i} d>0$. Here $v_{\perp}$ and $u_{\perp}$ are the magnitudes of the components of $\mathbf{v}$ and $\mathbf{u}$ perpendicular to the $z$ direction. It is known that the initial ion stream velocity $v_{B}$ is typically given by the ion sound speed $v_{B}=\left(k_{B} T_{c} / m\right)^{1 / 2}$, where $k_{B}$ is the Boltzmann constant, $T_{e}$ is the electron temperature of the bulk plasma, and $m$ is the ion mass (this is the Bohm sheath criterion; see [21]). The initial value for the potential is given by $\phi(\xi=0)=0$, as before.

By expanding the ion distribution function $\bar{f}$ and the potential $\phi$ in terms of the small parameter $\epsilon$ in the form $\bar{f}=\bar{f}_{0}+\epsilon \bar{f}_{1}+\cdots$ and $\phi=\phi_{0}+\epsilon \phi_{1}+\cdots$, we obtain from Eqs. (14) and (15) the following lowest-order equations for a collisionless sheath:

$$
\begin{aligned}
& u_{z} \frac{\partial \bar{f}_{0}}{\partial \zeta}-\frac{d \phi}{d \zeta} \frac{\partial \bar{f}_{0}}{\partial u_{z}}=0, \\
& \frac{d^{2} \phi_{0}}{d \xi^{2}}=-\int \bar{f}_{0}(\mathbf{u}, \zeta) d \mathbf{u} .
\end{aligned}
$$

With the use of the new independent variable

$$
\mathscr{E}=\frac{1}{2} u_{z}^{2}+\phi_{0}(\zeta)
$$

Eq. (17) becomes

$$
u_{z} \frac{\partial \bar{f}_{0}\left(u_{\perp}, \mathscr{E}, \operatorname{sgn} u_{z}, \zeta\right)}{\partial \zeta}=0
$$

and its solution with the initial condition (16) is given by $\bar{f}_{0}=\left\{\begin{array}{l}\frac{\delta\left(u_{\perp}\right)}{2 \pi u_{\perp}} \delta\left(\sqrt{2 \mathscr{E}}-u_{B}\right) \text { if } \operatorname{sgn} u_{z}=1 \text { and } \mathscr{E} \geq 0 \\ 0 \text { otherwise }\end{array}\right.$

Here $\operatorname{sgn} u_{z}$ denotes the sign of the function $u_{z}(\mathscr{E}, \xi)$, i.e., $\operatorname{sgn} u_{z}=u_{z} /\left|u_{z}\right|$ when $\left|u_{z}\right| \neq 0$. As shown in Eq. (6), the ion flux $\Gamma_{0}$ is independent of $z$. From the initial condition (16) and the lowest-order Boltzmann equation (17), therefore, we have

$$
\Gamma_{0}=n_{I} v_{B}=n_{0}(z) v_{0}(z),
$$

where $n_{0}$ is the density of the ballistic ions defined by $n_{0}=n_{I} \int \bar{f}_{0} d \mathbf{u}$ and $v_{0}$ is the ion stream velocity of the ballistic ions defined by the equation above. It is easy to check from Eq. (20) that the ion stream velocity is given by

$$
\frac{v_{0}}{\omega_{p i} d}=u_{0}(\zeta)=\sqrt{u_{B}^{2}-2 \phi_{0}(\zeta)} \text {. }
$$

The lowest-order potential drop $\phi_{0}$ may be calculated through substitution of Eq. (20) into Eq. (18), i.e.,

$$
\frac{d^{2} \phi_{0}}{d \zeta^{2}}=\frac{-u_{B}}{\sqrt{u_{B}^{2}-2 \phi_{0}}} .
$$

The exact, closed-form solution of Eq. (22) has recently been derived in [23], where it is shown that $\phi_{0}$ is a nonpositive $\left(\phi_{0} \leq 0\right)$ monotonically decreasing function for all $\xi \geq 0$. We note that the solution of Eq. (22) in the limit of $v_{B} \rightarrow 0$ with fixed ion flux $\Gamma_{0}=n_{I} v_{B}$ [or, equivalently, $u_{B} \rightarrow 0$ with $\left.\phi_{0} u_{B}^{2 / 3} \simeq O(1)\right]$ and the condition $E_{I}=0$ gives the collisionless Child-Langmuir law [24]

$$
\Phi=-6^{1 / 3} \frac{3}{4}\left(n_{I} v_{B} q / \epsilon_{0}\right)^{2 / 3}(m / q)^{1 / 3} z^{4 / 3}
$$

or

$$
-\phi_{0}=\frac{1}{2}\left(\frac{9 u_{B}}{2}\right)^{2 / 3} \xi^{4 / 3} .
$$

We now proceed to the first-order equations, which are given by

$$
\begin{aligned}
& u_{z} \frac{\partial \bar{f}_{1}}{\partial \zeta}-\frac{d \phi_{0}}{d \zeta} \frac{\partial \bar{f}_{1}}{\partial u_{z}}-\frac{d \phi_{1}}{d \zeta} \frac{\partial \bar{f}_{0}}{\partial u_{z}}=B^{+}-B^{-} \\
& \frac{d^{2} \phi_{1}}{d \zeta^{2}}=-\int \bar{f}_{1}(\mathbf{u}, \zeta) d \mathbf{u}
\end{aligned}
$$

where

$$
B^{+}=u \int\left[\frac{u^{\prime}}{u}\right]^{4} \bar{f}_{0}\left(\mathbf{u}^{\prime}\right) \frac{\sigma}{\sigma_{\text {tot }}} d^{2} \Omega
$$

and

$B^{-}=\left\{\begin{array}{l}u \frac{\delta\left(u_{\perp}\right)}{2 \pi u_{\perp}} \delta\left(\sqrt{2 \mathscr{E}}-u_{B}\right) \text { if } \operatorname{sgn} u_{z}=1 \text { and } \mathscr{E} \geq 0 \\ 0 \text { otherwise }\end{array}\right.$

The component $B^{+}$of the collision integral may be evaluated as follows: 


$$
\begin{aligned}
& B^{+}=\int_{V_{S}} \delta(\mathbf{u}) B^{+} d \mathbf{u} \\
& =\int_{V_{S}} d \mathbf{u}^{\prime} \frac{1}{4 \pi} \int u^{\prime} \bar{f}_{0}\left(\mathbf{u}^{\prime}\right) \sin \chi_{\mathrm{c} . \mathrm{m} .} d \chi_{\mathrm{c} . \mathrm{m} .} d \psi \\
& =\frac{1}{\pi} \int_{0}^{\infty} d u_{\perp}^{\prime} \int_{0}^{\infty} d u_{z}^{\prime} \int_{0}^{\pi} d \chi \int_{0}^{2 \pi} d \psi \delta(\mathbf{u}) 2 \pi u_{\perp}^{\prime} u^{\prime} \bar{f}_{0}\left(\mathbf{u}^{\prime}\right) \sin \chi \cos \chi \\
& =\left\{\begin{array}{lr}
\frac{1}{\pi} \int_{0}^{\infty} d u_{\perp}^{\prime} \int_{0}^{\infty} d u^{\prime} \int_{0}^{\pi / 2} d \theta \int_{0}^{2 \pi} d \varphi \delta(\mathbf{u}) \delta\left(u_{\perp}^{\prime}\right) \delta\left[\sqrt{u^{\prime 2}+2 \phi_{0}(\zeta)}-u_{B}\right] u^{\prime} \sin \theta \cos \theta \\
0 \text { otherwise } & \text { if } \cos \theta>0 \text { and } u^{\prime 2}+2 \phi_{0}(\zeta) \geq 0
\end{array}\right. \\
& = \begin{cases}\int_{0}^{\infty} u^{2} d u \int_{0}^{\pi / 2} \sin \theta d \theta \int_{0}^{2 \pi} d \varphi \delta(\mathbf{u}) \frac{1}{\pi u \cos \theta} \delta\left(\sqrt{u^{2} / \cos ^{2} \theta+2 \phi_{0}(\zeta)}-u_{B}\right) \\
0 \text { otherwise } & \text { if } \cos \theta>0 \text { and } \frac{u^{2}}{\cos ^{2} \theta}+2 \phi_{0}(\zeta) \geq 0\end{cases}
\end{aligned}
$$

where Eqs. (9), (10), (20), and the relations $u=u^{\prime} \cos \chi$ and $\left(u^{\prime} / u\right)^{3}=\partial\left(\mathbf{u}^{\prime}\right) / \partial(\mathbf{u})$ are used. Here $V_{S}$ is the entire velocity space, $\theta$ denotes the polar angle between $\mathbf{u}$ and the $z$ axis, and $\varphi$ denotes the azimuthal angle about the $z$ axis. It should be noted that, in deriving Eq. (28) from Eq. (27), we have used $\chi=\theta, \psi=\varphi$, and $u_{z}^{\prime}=u^{\prime}$ since $u_{\perp}^{\prime}=0$, and in deriving Eq. (29) from Eq. (28), we have used $u^{\prime}=u / \cos \theta$ [see Eq. (10)]. Carrying out the integration with respect to $\mathbf{u}$ in Eq. (28) and using $\cos \theta=u_{z} / u$, we obtain

$$
B^{+}=\left\{\begin{array}{l}
\frac{1}{\pi u_{z}} \delta\left(\sqrt{h}-u_{B}\right) \\
\quad \text { if } u_{z}>0 \text { and } h \equiv \frac{\left(u_{\perp}^{2}+u_{z}^{2}\right)^{2}}{u_{z}^{2}}+2 \phi_{0} \geq 0 \\
0 \text { otherwise . }
\end{array}\right.
$$

In order to solve the first-order equation (23), we again transform the variable $u_{z}$ to $\mathscr{E}$ defined in Eq. (19), so that the sum of the first two terms on the left-hand side of Eq. (23) becomes $u_{z} \partial \bar{f}_{1}\left(u_{\perp}, \mathscr{E}, \operatorname{sgn} u_{z}, \xi\right) / \partial \zeta$. We note that the domain of the function $\bar{f}_{1}$ is $u_{1} \geq 0, \mathscr{E}-\phi_{0}=\left(\frac{1}{2}\right) u_{z}^{2} \geq 0$, and $\zeta \geq 0$ (see Fig. 1). As shown in Eq. (10), the parallel

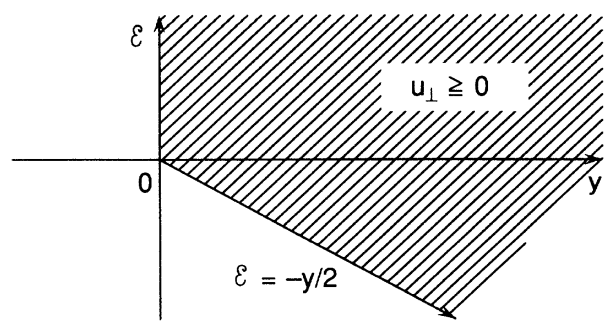

FIG. 1. The domain on which $\bar{f}_{1}$ is defined, where $y=-2 \phi_{0}(\zeta)$. velocities $u_{z}$ of scattered ions are positive (i.e., $0 \leq \chi \leq \pi / 2$ ) and, therefore, $\bar{f}_{1}=0$ for $\operatorname{sgn} u_{z}=-1$. The initial conditions for $\bar{f}_{1}$ with $\operatorname{sgn} u_{z}=1$ are then given by $\bar{f}_{1}=0$ on the boundary $\xi=0$ for $\mathscr{E} \geq 0$ and on the boundary $\mathscr{E}=\phi_{0}(\xi)$ for $\mathscr{E} \leq 0$, as indicated in Fig. 1. It is convenient to split $\bar{f}_{1}$ as $\bar{f}_{1}=\bar{f}_{1}^{+}+\bar{f}_{1}^{-}$in such a way that Eq. (23) may be written as

$$
u_{z} \frac{\partial \bar{f}_{1}^{+}}{\partial \zeta}=B^{+}
$$

and

or

$$
u_{z} \frac{\partial \bar{f}_{1}^{-}}{\partial \zeta}-\frac{d \phi_{1}}{d \zeta} \frac{\partial \bar{f}_{0}}{\partial u_{z}}=-B^{-}
$$

$$
\frac{\partial f_{1}^{+}}{\partial \zeta}=\left\{\begin{array}{l}
\frac{1}{\pi u_{z}^{2}} \delta\left(\sqrt{h}-u_{B}\right) \text { if } \operatorname{sgn} u_{z}=1 \text { and } h \geq 0 \\
0 \text { otherwise },
\end{array}\right.
$$

with $u_{z}^{2}(\mathscr{E}, \zeta)=2\left[\mathscr{E}-\phi_{0}(\zeta)\right]$ and

$$
\frac{\partial \bar{f}_{1}^{-}}{\partial \zeta}-\frac{1}{u_{z}} \frac{d \phi_{1}}{d \zeta} \frac{\partial \bar{f}_{0}}{\partial u_{z}}=\left\{\begin{array}{c}
-\frac{\delta\left(u_{\perp}\right)}{2 \pi u_{\perp}} \delta\left(\sqrt{2 \mathscr{E}}-u_{B}\right) \\
\quad \text { if } \operatorname{sgn} u_{z}=1 \text { and } \mathscr{E} \geq 0 \\
0 \text { otherwise },
\end{array}\right.
$$

where $u \delta\left(u_{\perp}\right)=u_{z} \delta\left(u_{\perp}\right)$ is used. The second term on the left-hand side of Eqs. (32) may be simplified as

$$
-\frac{1}{u_{z}} \frac{d \phi_{1}}{d \zeta} \frac{\partial \bar{f}_{0}}{\partial u_{z}}=\frac{d \phi_{1}}{d \zeta} \frac{\delta\left(u_{\perp}\right)}{2 \pi u_{\perp}} \frac{\delta\left(\sqrt{2 \mathscr{E}}-u_{B}\right)}{u_{B}\left(\sqrt{2 \mathscr{E}}-u_{B}\right)},
$$

where the relation $d \delta(x) / d x=-\delta(x) / x$ is used. The initial conditions for $\bar{f}$ and $\phi$ require that $\bar{f}_{1}^{+}=f_{1}^{-}=0$ and 
$\phi_{1}=0$ at $\zeta=0$. With these initial conditions, Eq. (32) is readily integrated and we obtain

$\bar{f}_{1}^{-}=-\frac{\delta\left(u_{\perp}\right)}{2 \pi u_{\perp}} \delta\left(\sqrt{2 \mathscr{E}}-u_{B}\right) \xi-\frac{\delta\left(u_{\perp}\right)}{2 \pi u_{\perp}} \frac{\delta\left(\sqrt{2 \mathscr{E}}-u_{B}\right)}{u_{B}\left(\zeta 2 \mathscr{E}-u_{B}\right)} \phi_{1}(\zeta)$

if $\operatorname{sgn} u_{z}=1$ and $\mathscr{E} \geq 0$, and $\bar{f}_{1}^{-}=0$ otherwise.

We now integrate Eq. (31). Since $\phi_{0}(\zeta)$ is a monotonically decreasing function, we transform the variable $\zeta$ to $y$ by

$$
y=-2 \phi_{0}(\zeta)
$$

where $y \geq 0$ and $y \geq-2 \mathscr{E}$. Then Eq. (31) becomes

$$
\frac{\partial f_{1}^{+}}{\partial y}=-\frac{\delta\left(\sqrt{h}-u_{B}\right)}{2 \phi_{0}^{\prime} \pi(2 \mathscr{E}+y)}
$$

if $\operatorname{sgn} u_{z}=1$ and $h \geq 0$, otherwise $\partial f_{1}^{+} / \partial y=0$. Here $\phi_{0}^{\prime}=d \phi_{0} / d \zeta$ evaluated at $\zeta=\zeta(y)=\phi_{0}^{-1}(-y / 2)$, and the function $h$, defined in Eq. (31), may be written in terms of $\mathscr{E}, u_{1}$, and $y$ as

$$
h=2\left(u_{\perp}^{2}+\mathscr{E}\right)+\frac{u_{\perp}^{\prime}}{2 \mathscr{E}+y},
$$

which is a monotonically decreasing function of $y(>-2 \mathscr{E})$. In integrating Eq. (31), we consider three mutually disjoint regions, namely, $\mathscr{E} \geq 0,0>\mathscr{E} \geq-u_{\perp}^{2}$, and $-u_{\perp}^{2}>\mathscr{E}$.

In the case $\mathscr{E} \geq 0$, the range of the function $h$ is

$$
\begin{aligned}
0 \leq 2\left(u_{\perp}^{2}+\mathscr{E}\right)<h \leq h_{0}\left(\mathscr{E}, u_{\perp}\right) & \equiv h\left(\mathscr{E}, u_{\perp}, y=0\right) \\
& =2\left(u_{\perp}^{2}+\mathscr{E}\right)+u_{\perp}^{4} / 2 \mathscr{E}
\end{aligned}
$$

for fixed $\mathscr{E}$ and $u_{\perp}$. Therefore, if

$$
2\left(u_{\perp}^{2}+\mathscr{E}\right)<u_{B}^{2} \leq h_{0}\left(\mathscr{E}, u_{\perp}\right)=2\left(u_{\perp}^{2}+\mathscr{E}\right)+\frac{u_{\perp}^{4}}{2 \mathscr{E}},
$$

integration of Eq. (36) over $y$ from $y=0$ yields

$$
f_{1}^{+}=\frac{u_{B}}{-\pi \phi_{0}^{\prime}\left(\zeta_{c}\right)\left[u_{B}^{2}-2\left(u_{\perp}^{2}+\mathscr{E}\right)\right]}
$$

when $h\left(\mathscr{E}, u_{1}, y\right) \leq u_{B}^{2}$, and $f_{1}^{+}=0$ otherwise. If the condition (37) is not satisfied, then also $f_{1}^{+}=0$. In Eq. (38), $\zeta_{c}=\zeta_{c}\left(u_{\perp}, \mathscr{E}\right)$ denotes the $\zeta$ value that satisfies $h=u_{B}^{2}$, or $\zeta_{c}=\zeta\left(y_{c}\right)$ with

$$
y_{c}=-2 \mathscr{E}+\frac{u_{\perp}^{4}}{u_{B}^{2}-2\left(u_{\perp}^{2}+\mathscr{E}\right)} .
$$

Physically, the variables $u_{\perp}$ and $\mathscr{E}$ are constants of motion of the system and the condition $h=u_{B}^{2}$ states that the ion having such $u_{\perp}$ and $\mathscr{E}$ satisfies the collision condition $u=u^{\prime} \cos \theta$ [Eq. (10)] at the position specified by the potential $\phi_{0}=-y_{c} / 2$ of Eq. (39) or, in other words, at $\xi=\zeta_{c}\left(u_{1}, \mathscr{E}\right)$. In the case $-u_{\perp}^{2} \leq \mathscr{E}<0$, the function $h$ has the range $0 \leq 2\left(u_{\perp}^{2}+\mathscr{E}\right)<h<\infty$. Therefore, if $\left(2 u_{\perp}^{2}\right.$ $+\mathscr{E})<u_{B}^{2}$, then integration of Eq. (31) over $y$ from $y=-2 \mathscr{E}$ yields the solution $f_{1}^{+}$given by Eq. (38). Other-
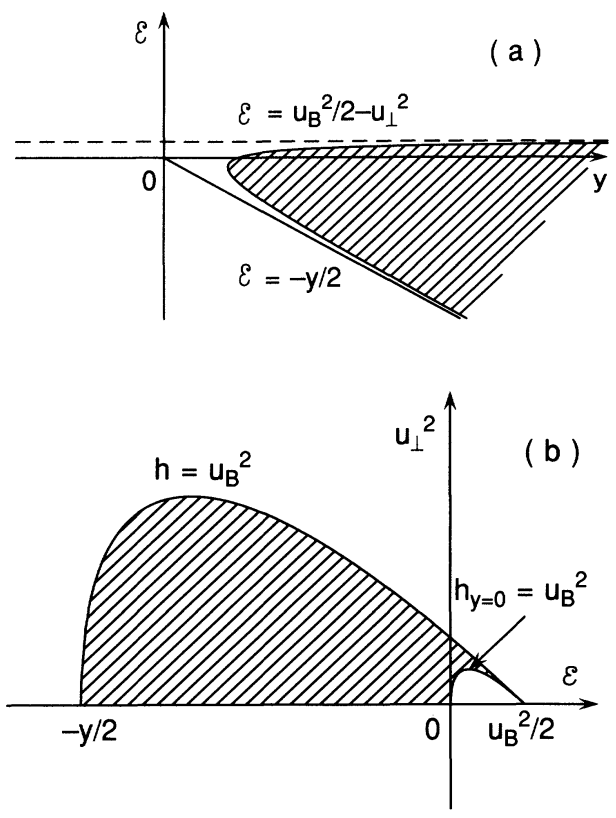

FIG. 2. The region on which $\bar{f}_{1}^{+}$is given by Eq. (38): (a) the region on the $y-\mathscr{E}$ plane with a fixed $u_{1}^{2}$, and (b) the region on the $\mathscr{E}-u_{\perp}^{2}$ plane with a fixed $y$. Outside this hatched region, $\bar{f}_{1}^{+}=0$.

wise $f_{1}^{+}=0$. In the case $\mathscr{E}<-u_{\perp}^{2}$, since $\left(2 u_{\perp}^{2}+\mathscr{E}\right)<h<\infty$ and $2\left(u_{\perp}^{2}+\mathscr{E}\right) \leq 0$, the solution $f_{1}^{+}$is given by Eq. (38) for any $u_{B}^{2}$, even in the case $h \leq 0$. In summary, the region where the solution $f_{1}^{+}$is expressed by Eq. (38) is given by the hatched region in Figs. 2(a) and $2(\mathrm{~b})$.

\section{ANGULAR DISTRIBUTIONS}

Having derived the ion distribution function in the previous section, we now calculate the angular distributions of the ion flux incident on the electrodes and of the ion density at the electrodes. The angular distribution of the ion flux, in particular, is of practical importance since it determines the anisotropy of the process plasma. In Sec. IV A a general formulation giving the angular distributions of the ion flux and the ion density is presented. The cases of a constant electric field and a self-consistent electric field are discussed in Secs. IV B and IV C, respectively. The ratio of the ballistic ion flux to the scattered ion flux and the ratio of the ballistic ion density to the scattered ion density are calculated in Sec. IV D.

\section{A. General formulation}

We first calculate the angular distribution of the ion density,

$$
\begin{aligned}
F_{\theta}\left(\theta_{1} z\right) & =\int_{0}^{2 \pi} d \varphi \int_{0}^{\infty} v^{2} d v f(v, \theta, z) \sin \theta \\
& =\frac{2 \pi d n_{I} \sin \theta}{\lambda_{\mathrm{MFP}}} \int_{0}^{\infty} d u u^{2} \bar{f}_{1}^{+}\left(\mathscr{E}, u_{\perp}, \xi\right),
\end{aligned}
$$


and of the ion flux,

$$
\begin{aligned}
\Gamma_{\theta}(\theta, z) & =\int_{0}^{2 \pi} d \varphi \int_{0}^{\infty} v^{2} d v v_{z} f(v, \theta, z) \sin \theta \\
& =\frac{2 \pi n_{I} \omega_{p l} d^{2} \sin \theta \cos \theta}{\lambda_{\mathrm{MFP}}} \int_{0}^{\infty} d u u^{3} \bar{f}_{1}^{+}\left(\mathscr{E}, u_{\perp}, \zeta\right)
\end{aligned}
$$

where

$$
\mathscr{E}=\frac{1}{2} u^{2} \cos ^{2} \theta+\phi_{0}(\zeta) \text { and } u_{\perp}=u \sin \theta
$$

In this section, we are concerned only with angular distributions for $\theta>0$ and do not count ballistic ions $(\theta=0)$ that do not suffer a collision. We note that $\bar{f}_{1}^{-}$given in Eq. (34) gives only a ballistic contribution to $F_{\theta}$ and $\Gamma_{\theta}$, because of the factor $\delta\left(u_{\perp}\right)$. The ratios of the scattered ion density and flux, given by Eqs. (40) and (41), respectively, to those of ballistic ions are also of practical importance, and will be discussed later in Sec. IV D.

In order to carry out the integration of Eqs. (40) and (41), we need to determine the range of the integration variable $u$ for which $\bar{f}_{1}^{+}$is given by Eq. (38). As discussed in the previous section, $\bar{f}_{1}^{+}$is given by Eq. (38) when

$$
h=2\left(u_{\perp}^{2}+\mathscr{E}\right)+\frac{u_{\perp}^{4}}{2 \mathscr{E}+y} \leq u_{B}^{2}
$$

and

$$
2\left(u_{1}^{2}+\mathscr{E}\right)+\frac{u_{1}^{4}}{2 \mathscr{E}} \geq u_{B}^{2}
$$

on the domain $y \geq 0, y \geq-2 \mathscr{E}$, and $u_{1} \geq 0$. From Eq. (42) and the inequality (43), we obtain the condition

$$
u^{2} \leq\left(u_{B}^{2}+y\right) \cos ^{2} \theta
$$

Substituting Eq. (42) into the inequality (44) yields the condition

$$
u^{4}-\left(2 y+u_{B}^{2} \cos ^{2} \theta\right) u^{2}+\left(y+u_{B}^{2}\right) y \geq 0 .
$$

The discriminant of this quadratic equation for $u^{2}$ is given by

$$
\begin{aligned}
D & =\left(2 y+u_{B}^{2} \cos ^{2} \theta\right)^{2}-4\left(y+u_{B}^{2}\right) y \\
& =u_{B}^{2}\left(u_{B}^{2} \cos ^{4} \theta-4 y \sin ^{2} \theta\right) .
\end{aligned}
$$

Therefore, the range of $u$ is given as follows:

if $\frac{u_{B}^{2}}{4 y}<\frac{\sin ^{2} \theta}{\cos ^{4} \theta}(\Longrightarrow D<0)$, then $0 \leq u^{2} \leq\left(u_{B}^{2}+y\right) \cos ^{2} \theta$ and

if $\frac{u_{B}^{2}}{4 y} \geq \frac{\sin ^{2} \theta}{\cos ^{4} \theta}(\Longleftrightarrow D \geq 0)$,

then

$$
0 \leq u^{2} \leq y+\frac{u_{B}^{2}}{2} \cos ^{2} \theta-\frac{1}{2} \sqrt{D}
$$

and

$$
y+\frac{u_{B}^{2}}{2} \cos ^{2} \theta+\frac{1}{2} \sqrt{D} \leq u^{2} \leq\left(u_{B}^{2}+y\right) \cos ^{2} \theta .
$$

It is easy to show that

$$
\left(u_{B}^{2}+y\right) \cos ^{2} \theta \geq y+\left(u_{B}^{2} / 2\right) \cos ^{2} \theta+\sqrt{D} / 2
$$

for all $y \geq 0$ and $\theta$. It should be noted that the term $u_{B}^{2} / y=m u_{B}^{2} / 2 q\left|\Phi_{0}\right|$ denotes the ratio of the initial ion kinetic energy to the zeroth-order potential energy $\Phi_{0}$ at $z=\zeta d$ and typically takes a small value.

It is an interesting observation to write Eq. (39) as

$$
y_{c}=\frac{u^{4}-\left(2 y+u_{B}^{2} \cos ^{2} \theta\right) u^{2}+\left(y+u_{B}^{2}\right) y}{\left(u_{B}^{2}+y\right)-\left(1+\sin ^{2} \theta\right) u^{2}},
$$

where the relation

$$
u_{B}^{2}-2\left(u_{\perp}^{2}+\mathscr{E}\right)=\left(u_{B}^{2}+y\right)-u^{2}\left(1+\sin ^{2} \theta\right)
$$

is used. From the inequality (43), it is obvious that the denominator of Eq. (49) [or equivalently the denominator of Eq. (39)] is positive, so that the condition (46) [or equivalently Eq. (44)] requires that $y_{c} \geq 0$. On the other hand, with the use of the above expression for $y_{c}$, it is easy to show that the condition $y_{c} \leq y$ is equivalent to the condition (45) [or equivalently (44)]. Since the monotonicity of the potential $\phi_{0}(\xi)=-y / 2$ implies that the inequalities $y_{c} \geq 0$ and $y_{c} \leq y$ indicate that the collision location $\zeta_{c}$ satisfies $\zeta_{c} \geq 0$ and $\zeta_{c} \leq \zeta$, respectively, the conditions (45) and (46) ensure that the ions collide where they are allowed to collide in our model, i.e., $0 \leq \zeta_{c}<\zeta$. (Note that we are observing the ion distribution function at $\zeta$ and, in the low-collisionality limit, the ions that have collided with neutrals must have collided before reaching the position $\zeta$ if the ions have the same or larger mass than the neutrals $m \geq M$ [5].) A similar physical interpretation of the conditions (47) and (48) is discussed in the Appendix.

In the case

$$
u_{B}^{2} / 4 y=m u_{B}^{2} / 8 q\left|\Phi_{0}\right|<\sin ^{2} \theta / \cos ^{4} \theta
$$

where the inequality (47) holds, therefore, the angular distribution of the ion density is given by

$$
F_{\theta}(\theta, z)=\frac{2 \pi d n_{I} \sin \theta}{\lambda_{\mathrm{MFP}}} \int_{0}^{\sqrt{u_{B}^{2}+y} \cos \theta} \frac{u_{B} u^{2} d u}{-\pi \phi_{0}^{\prime}\left(\zeta_{c}\right)\left[\left(u_{B}^{2}+y\right)-u^{2}\left(1+\sin ^{2} \theta\right)\right]},
$$

where the relation

$$
u_{B}^{2}-2\left(u_{\perp}^{2}+\mathscr{E}\right)=\left(u_{B}^{2}+y\right)-u^{2}\left(1+\sin ^{2} \theta\right)
$$


is used again. Similarly, the angular distribution of the ion flux is given by

$$
\Gamma_{\theta}(\theta, z)=\frac{2 \pi n_{I} \omega_{p i} d^{2} \sin \theta \cos \theta}{\lambda_{\mathrm{MFP}}} \int_{0}^{\sqrt{u_{B}^{2}+y} \cos \theta} \frac{u_{B} u^{3} d u}{-\pi \phi_{0}^{\prime}\left(\zeta_{c}\right)\left[\left(u_{B}^{2}+y\right)-u^{2}\left(1+\sin ^{2} \theta\right)\right]} .
$$

For the small angles satisfying $u_{B}^{2} / 4 y \geq \sin ^{2} \theta / \cos ^{4} \theta$, the range of integration of Eqs. (50) and (51) needs to be changed according to the inequality (48).

We note that, in deriving the ion distribution function given in Eq. (38) and, subsequently, the angular distribution functions given in Eqs. (50) and (51), the potential $\phi_{0}(\xi)$ is treated as a given function; in other words, for the distribution function $\bar{f}_{1}^{+}$to have the form (38) does not mathematically require that the field equation (18) [or, equivalently Eq. (22)] be satisfied by $\phi_{0}$. In order to obtain a physically self-consistent solution, of course, the potential $\phi_{0}$ used in those formulas needs to satisfy Eq. (18). However, it is still instructive to use a simpler form for the potential $\phi_{0}$, which does not necessarily satisfy Eq. (18), in order to further proceed with the calculations and obtain simple mathematical formulas for various important quantities. In the following subsection, we shall assume a constant electric field, $\phi_{0}^{\prime}=$ const, to execute integration of Eqs. (50) and (51).

\section{B. Constant fields}

If the electric field is constant, then the term $-\phi_{0}^{\prime}=\bar{E}_{I}$ may be taken outside of the integration and we have

$$
\begin{aligned}
F_{\theta}(\theta, z)= & \frac{2 d n_{I} u_{B}\left(u_{B}^{2}+y\right)^{1 / 2}}{\lambda_{\mathrm{MFP}}} \\
& \times \int_{0}^{\cos \theta} \frac{\sin \theta \xi^{2} d \xi}{-\phi_{0}^{\prime}\left(\xi_{c}\right)\left[1-\left(1+\sin ^{2} \theta\right) \xi^{2}\right]} \\
=\frac{d n_{I}}{\lambda_{\mathrm{MFP}}} & \frac{u_{B}\left[u_{B}^{2}-2 \phi_{0}(\xi)\right]^{1 / 2}}{2 \bar{E}_{I}} \mathcal{F}_{\theta}(\theta),
\end{aligned}
$$

where

$$
\begin{aligned}
\mathscr{F}_{\theta}(\theta)=\frac{2 \sin \theta}{1+\sin ^{2} \theta}[ & \frac{1}{\sqrt{1+\sin ^{2} \theta}} \\
& \left.\times \ln \left(\frac{1+\sqrt{1-\sin ^{4} \theta}}{1-\sqrt{1-\sin ^{4} \theta}}\right]-2 \cos \theta\right] .
\end{aligned}
$$

In Eq. (52), $\xi=u / \sqrt{u_{B}^{2}+y}$ with $y=-2 \phi_{0}(\xi)$ is used. Since we are concerned with the case of small initial velocities where $\frac{1}{2} m v_{B}^{2}<q\left|\Phi_{0}\right|$ or $u_{B}^{2} / 4 y=m v_{B}^{2} /$ $8 q\left|\Phi_{0}\right| \ll 1$, Eq. (52) gives the angular distribution for most of $\theta>0$ correctly in the weak-collisionality limit. If $u_{B}^{2} / 4 y<<1$ holds, Eq. (52) may be further simplified with the use of $u_{B}^{2} \ll-\phi_{0}=\bar{E}_{I} \xi$ with

$$
\bar{E}_{I}=E(z) /\left(q d n_{I} / \epsilon_{0}\right)=E_{I} /\left(q d n_{I} / \epsilon_{0}\right)
$$

and given in dimensional form by

$$
F_{\theta}(\theta, z)=n_{0}(z) \frac{z}{\lambda_{\mathrm{MFP}}} \mathscr{F}_{\theta}(\theta),
$$

where we use the fact that $n_{I} u_{B}=n_{0}(z)\left[u_{B}^{2}-2 \phi_{0}(\zeta)\right]^{1 / 2}$. Here the condition $u_{B}^{2} / 4 y \ll 1$ requires that the range of $z$ in which the expression (54) holds be given by

$$
\frac{m v_{B}^{2}}{8 q E_{I} \lambda_{\mathrm{MFP}}} \ll \frac{z}{\lambda_{\mathrm{MFP}}}(\ll<1) .
$$

In most plasma-processing applications, the angular distribution of the ion flux is of more practical interest [20] than the angular distribution of the ion density discussed above. If the electric field is constant, then, as in Eq. (52), the integration of Eq. (51) can be easily performed, and we obtain

$$
\begin{aligned}
\Gamma_{\theta}(\theta, z)= & \frac{2 n_{I} \omega_{p i} d^{2} u_{B}\left(u_{B}^{2}+y\right)}{\lambda_{\mathrm{MFP}}} \\
& \times \int_{0}^{\cos \theta} \frac{\sin \theta \cos \theta \xi^{3} d \xi}{-\phi_{0}^{\prime}\left(\xi_{c}\right)\left[1-\left(1+\sin ^{2} \theta\right)^{2}\right]} \\
= & \frac{d}{\lambda_{\mathrm{MFP}}} \Gamma_{0} \frac{\left[u_{B}^{2}-2 \phi_{0}(\xi)\right]}{\bar{E}_{I}} g_{\theta}(\theta),
\end{aligned}
$$

where $\Gamma_{0}=n_{I} v_{B}$ and

$$
\mathcal{S}_{\theta}(\theta)=\frac{2 \sin \theta \cos \theta}{\left(1+\sin ^{2} \theta\right)^{2}}\left(4 \ln \frac{1}{\sin \theta}+\sin ^{4} \theta-1\right),
$$

which gives the profile of the ion-flux angular distribution. As in Eq. (52), $\xi=u / \sqrt{u_{B}^{2}+y}$. If the initial velocity $v_{B}$ is sufficiently small, so that the condition $u_{B}^{2} / 4 y \ll 1$ [or Eq. (55)] is satisfied, then Eq. (56) gives the angular distribution for most of $\theta>0$ correctly, as indicated by inequality (47). In this case, as in the case of Eq. (52), Eq. (56) may be further simplified with the use of $u_{B}^{2} \ll-\phi_{0}=\bar{E}_{I} \xi$ and given in dimensional form by

$$
\Gamma_{\theta}(\theta, z)=\frac{\Gamma_{0} z}{\lambda_{\mathrm{MFP}}} g_{\theta}(\theta) .
$$

Thus, $\Gamma_{\theta}$ is seen to be independent of the electric-field strength $E_{I}$ in the case of a constant electric field.

Figures 3(a) and 3(b) show angular distributions of the ion density and the ion flux, respectively, in the case of a constant electric field. Here the solid lines represent the theoretical profiles $\mathfrak{F}_{\theta}(\theta)$ for Fig. 3(a) and $\mathcal{S}_{\theta}(\theta)$ for Fig. 3 (b) given in Eqs. (53) and (57), respectively, and the histograms are obtained from the Monte Carlo simulations. The Monte Carlo simulation code used here is capable of handling both constant and self-consistent electric fields; the details of this code are given in [5]. An excellent agreement between the theoretically predicted profiles and the Monte Carlo data is evident in Fig. 3. The simu- 
lation parameters are $d / \lambda_{\mathrm{MFP}}=0.14, E_{I}=4.1 \times 10^{-2}$, $u_{B}=4.2 \times 10^{-5}$,

$$
u_{B}^{2} / 4 y=m v_{B}^{2} / 8 q E_{I} d=5.1 \times 10^{-7},
$$

and $\zeta=z / d=1$. In Figs. 3(a) and 3(b), the profiles are normalized in such a way that the areas under the curves and the histograms representing scattered ions are equal to unity. This requires use of the following normalization factors:

$$
\begin{aligned}
& \mathscr{F}_{0}=\int_{0}^{\pi / 2} \mathscr{F}_{\theta}(\theta) d \theta \simeq 1.39, \\
& \mathcal{S}_{0}=\int_{0}^{\pi / 2} \mathcal{S}_{\theta}(\theta) d \theta=1.0 .
\end{aligned}
$$

Note also that the ballistic components of the Monte Carlo simulations in these figures, at $\theta=0$, which correspond to $\delta$ functions, are truncated at $F_{\theta}=\Gamma_{\theta}=3$ or by the frames of the figures.
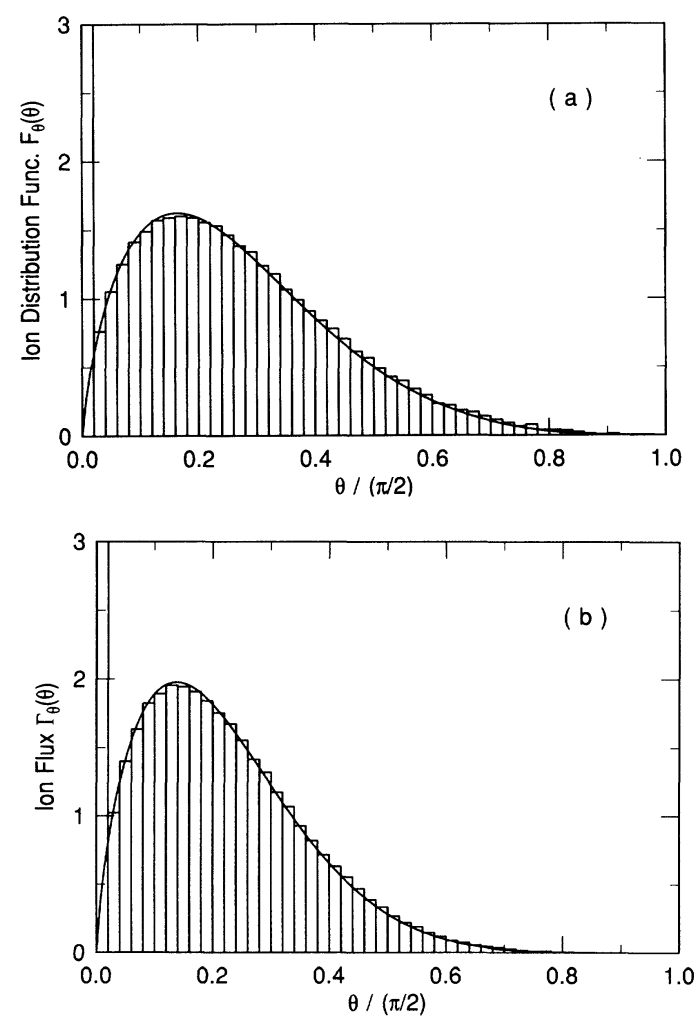

FIG. 3. The angular distributions (a) of the ion density and (b) of the ion flux in the case of a constant electric field obtained from the Monte Carlo simulations (histogram), Eq. (53) [the solid curve in (a)], and Eq. (57) [the solid curve in (b)]. The ballistic ion component, represented by the first bin at $\theta=0$, is truncated. The magnitudes of $F_{\theta}$ and $\Gamma_{\theta}$ are normalized as described in the text. The dimensionless parameters for these results are $d / \lambda_{\mathrm{MFP}}=0.14, u_{B}=4.2 \times 10^{-5}, \bar{E}_{I}=4.1 \times 10^{-2}$, and $\xi=1$.

\section{Self-consistent fields}

In the case of self-consistent electric fields, where the potential $\phi_{0}$ is obtained by solving Eq. (22), the electric field $\phi_{0}^{\prime}$ is no longer independent of $\zeta$ and the term $\phi_{0}^{\prime}\left(\zeta_{c}\right)$ becomes a function of $u$ and $\theta$. The angular distributions $F_{\theta}(\theta, z)$ and $\Gamma_{\theta}(\theta, z)$ then need to be calculated directly from Eqs. (50) and (51) with knowledge of the dependence of $\phi_{0}^{\prime}$ on $u$ and $\theta$. As discussed in Sec. III, $\phi_{0}^{\prime}\left(\xi_{c}\right)$ is the derivative of $\phi_{0}$ evaluated at $\xi=\zeta_{c}$, where the potential $\phi_{0}$ satisfies $-2 \phi_{0}=y_{c}$ with $y_{c}$ given in Eq. (39) or Eq. (49). As we shall presently show, $\phi_{0}$ may be expressed as an explicit function of $u$ and $\theta$.

Multiplying Eq. (22) by $d \phi_{0} / d \xi$ and integrating with respect to $\zeta$, we obtain

$$
\frac{1}{2}\left(\frac{d \phi_{0}}{d \zeta}\right)^{2}=-u_{B} \int_{0}^{\phi_{0}} \frac{d \phi_{0}}{\sqrt{u_{B}^{2}-2 \phi_{0}}}+\frac{1}{2} \bar{E}_{I}^{2},
$$

where the initial condition $d \phi_{0} / d \xi=\bar{E}_{I}$ is used. Carrying out this integration and substituting $\phi_{0}=-y_{c} / 2$ yields

$$
\phi_{0}^{\prime}\left(\zeta_{c}\right)=-\left[2\left(u_{B} \sqrt{u_{B}^{2}+y_{c}}+u_{B}^{2} K\right)\right]^{1 / 2},
$$

where $K=\bar{E}_{I}^{2} / 2 u_{B}^{2}-1(>-1)$. It is shown in [23] that the potential $\phi_{0}$ is a weak function of $K$ for realistic values of $K\left(-1<K<\frac{1}{3}\right)$. The function $y_{c}$ is given by Eq. (49) and satisfies

$$
u_{B}^{2}+y_{c}=\frac{\left[\left(u_{B}^{2}+y\right)-u^{2}\right]^{2}}{\left(u_{B}^{2}+y\right)-\left(1+\sin ^{2} \theta\right) u^{2}} .
$$

Equation (61), together with Eq. (49) [or Eq. (62)], gives a closed-form expression for the dependence of $\phi_{0}^{\prime}\left(\zeta_{c}\right)$ on $u$ and $\theta$.

Introducing

$$
\xi=\frac{u}{u_{\max }}, \quad \alpha=\frac{u_{B}}{u_{\max }} \text { with } u_{\max }=\sqrt{u_{B}^{2}+y},
$$

we may write $-\phi_{0}^{\prime}\left(\zeta_{c}\right)=\sqrt{2 \alpha} u_{\max } g(\xi)$, where

$g(\xi)=\left\{\sqrt{\left(1-\xi^{2}\right)^{2} /\left[1-\left(1+\sin ^{2} \theta\right) \xi^{2}\right]}+\alpha K\right\}^{1 / 2}$.

From Eqs. (50) and (51), the angular distribution of the ion density is then given by

$$
F_{\theta}(\theta, z)=n_{I} \frac{d}{\lambda_{\mathrm{MFP}}} \frac{u_{B}}{\sqrt{2 \alpha}} \mathscr{F}_{\theta}^{\mathrm{sc}}(\theta ; \alpha, K)
$$

and that of the ion flux is given by

$$
\Gamma_{\theta}(\theta, z)=\Gamma_{0} \frac{d}{\lambda_{\mathrm{MFP}}} \frac{u_{B}}{\sqrt{2 \alpha^{3}}} \mathcal{G}_{\theta}^{\mathrm{sc}}(\theta ; \alpha, K),
$$

where, in the case of a self-consistent field, we define

$$
\begin{aligned}
& \mathcal{F}_{\theta}^{\mathrm{sc}}(\theta ; \alpha, K)=2 \sin \theta \int_{I(\alpha)} \frac{\xi^{2} d \xi}{g(\xi)\left[1-\left(1+\sin ^{2} \theta\right) \xi^{2}\right]}, \\
& \mathcal{G}_{\theta}^{\mathrm{sc}}(\theta ; \alpha, K)=2 \sin \theta \cos \theta \int_{I(\alpha)} \frac{\xi^{3} d \xi}{g(\xi)\left[1-\left(1+\sin ^{2} \theta\right) \xi^{2}\right]} .
\end{aligned}
$$


Here the range of integration $I(\alpha)$ is given as follows: from the inequality (47),

$$
\begin{aligned}
& \text { when } \alpha<\frac{2 \sin \theta}{\left(1+\sin ^{2} \theta\right)}\left[-\frac{u_{B}^{2}}{4 y}<\frac{\sin ^{2} \theta}{\cos ^{4} \theta}\right), \\
& I(\alpha)=\{0 \leq \xi \leq \cos \theta\} .
\end{aligned}
$$

Although $u_{B}^{2} / 4 y$ is generally small and the angular distributions for most values of $\theta$ are given by the integration over $I(\alpha)$ above, accurate accounts of small-angle distributions must be given by a different integral range $I(\alpha)$. From the inequality (48), we have

when $\alpha \geq \frac{2 \sin \theta}{\left(1+\sin ^{2} \theta\right)}\left[\Longleftarrow \frac{u_{B}^{2}}{4 y} \geq \frac{\sin ^{2} \theta}{\cos ^{4} \theta}\right)$,

$I(\alpha)=\left\{\begin{array}{l}0 \leq \xi \leq\left[\left(1-\alpha^{2}\right)+\frac{\alpha^{2}}{2} \cos ^{2} \theta-\hat{D}^{1 / 2} / 2\right]^{1 / 2} \\ {\left[\left(1-\alpha^{2}\right)+\frac{\alpha^{2}}{2} \cos ^{2} \theta+\widehat{D}^{1 / 2} / 2\right]^{1 / 2} \leq \xi \leq \cos \theta}\end{array}\right.$

Here

$$
\widehat{D}=\alpha^{2}\left[\alpha^{2} \cos ^{4} \theta-4\left(1-\alpha^{2}\right) \sin ^{2} \theta\right] .
$$

We note that the functions $\mathcal{F}_{\theta}^{\text {sc }}$ and $\mathcal{G}_{\theta}^{\text {sc }}$ depend on $K$ through the term $\alpha K$ in the function $g(\xi)$ of Eq. (64). Since the value $\alpha$ is typically small and the dependence of the potential $\phi_{0}$ on the parameter $K$ is known to be weak [23], the parameter dependence of $\mathcal{F}_{\theta}^{\text {sc }}$ and $\mathcal{S}_{\theta}^{\text {sc }}$ on $K$ is also weak.

Figures 4(a) and 4(b) show the angular distributions of the ion density and the ion flux, respectively: the histograms are obtained from the Monte Carlo simulations with a self-consistent electric field [5], the solid curves are obtained by numerical quadrature from Eqs. (67) and (68), and the dashed curves represent $\mathscr{F}_{\theta}(\theta)$ in Eq. (53) and $S_{\theta}(\theta)$ in Eq. (57). As in Fig. 3, the magnitudes of the distributions are normalized in such a way that the area under each curve is equal to unity, and the ballistic component is truncated. The parameters used in these calculations are: $d / \lambda_{\mathrm{MFP}}=0.14, \bar{E}_{I}=5.1 \times 10^{-3}, u_{B}=1.0$ $\times 10^{-2}$, and $\xi=z / d=1$. In other words, $u_{B}^{2} / 4 y$ $=2.1 \times 10^{-4}, \alpha=u_{B} / u_{\max }=2.9 \times 10^{-2}, K=-0.75$, and $\theta_{c}=0.015$, where $\theta_{c}$ denotes the angle where the two different regions given by inequalities (47) and (48) meet, i.e.,

$$
\frac{u_{B}^{2}}{4 y}=\frac{\sin ^{2} \theta_{c}}{\cos ^{4} \theta_{c}} \text { or } \alpha=\frac{2 \sin \theta_{c}}{\left(1+\sin ^{2} \theta_{c}\right)} .
$$

In dimensional quantities, these calculations correspond to an Ar discharge with assumed total ion-neutralspecies collision cross section $\sigma_{\text {tot }}=5.0 \times 10^{-15} \mathrm{~cm}^{2}[13$, 14], gas pressure $p_{g}=10 \mathrm{mTorr}$, neutral gas number density $n_{g}=2.7 \times 10^{14} \mathrm{~cm}^{-3}$, ionization fraction $n_{i} / n_{g}$ $=1.0 \times 10^{-4}$, sheath thickness $d=1 \mathrm{~mm}$, mean free path $\lambda_{\mathrm{MFP}}=7.3 \mathrm{~mm}$, and sheath potential drop $-\Phi=30 \mathrm{~V}$. Good agreement between the simulation results and the theoretical expressions [Eqs. (67) and (68)-solid curves] is clearly seen in Figs. 4, whereas the theoretical models based on the constant-field assumption [Eqs. (53) and (57) — dashed curves] predict distributions shifted slightly towards larger angles.

Since the analytic form of the ion distribution function $f$ obtained in Sec. III above is based on the assumption of weak collisionality $\left(d / \lambda_{\mathrm{MFP}}<<1\right)$, the question arises as to how large $d / \lambda_{\text {MFP }}$ may become before the "real" angular distributions (for finite $d / \lambda_{\mathrm{MFP}}$ ) begin to deviate appreciably from the analytic expressions derived above. Figure 5 shows a comparison of the analytic expression for the ion-flux angular distribution $\mathcal{S}_{\theta}^{\text {sc }}(\theta)$ (solid curves) with the self-consistent Monte Carlo simulation data for various levels of collisionality: $d / \lambda_{\mathrm{MFP}}=0.1,1.0,2.0$, and 4.0. As in Fig. 4(b), the dashed curves represent the angular distribution $S_{\theta}(\theta)$ for the constant-field approxi-
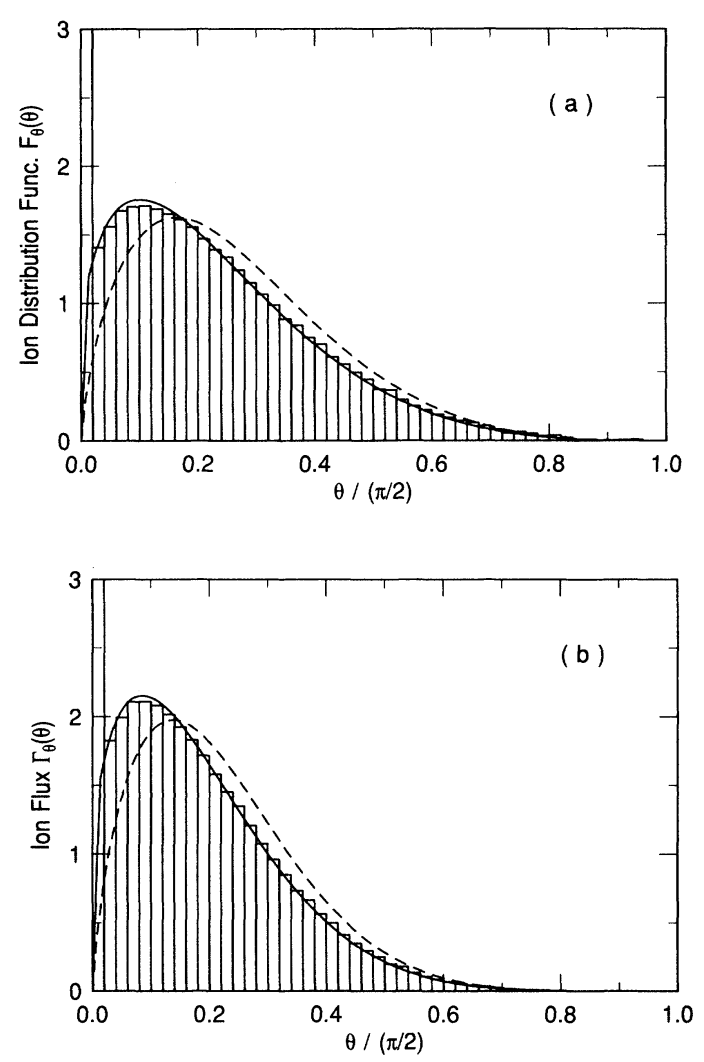

FIG. 4. The angular distributions (a) of the ion density and (b) of the ion flux in the case of a self-consistent electric field obtained from the Monte Carlo simulations (histogram), Eq. (67) [the solid curve in (a)], and Eq. (68) [the solid curve in (b)]. For comparison, the formulas for the constant-field approximation [Eq. (53) for (a) and Eq. (57) for (b)] are also presented as dashed lines. The ballistic ion component, represented by the first bin at $\theta=0$, is truncated. The magnitudes of $F_{\theta}$ and $\Gamma_{\theta}$ are normalized as in Fig. 3. The dimensionless parameters used here are $d / \lambda_{\mathrm{MFP}}=0.14, u_{B}=1.0 \times 10^{-2}, \bar{E}_{I}=5.1 \times 10^{-3}$, and $\xi=1$. 
mation Eq. (57). It is evident from Figs. 5(a) and 5(b) that the analytical expression $\mathcal{S}_{\theta}^{\text {sc }}(\theta)$ is a reasonably good approximation for collisionality $d / \lambda_{\text {MFP }}$ up to almost 1.0 , whereas, as expected, the discrepancy between the analytic expression for $g_{\theta}^{\text {sc }}(\theta)$ and the simulation results becomes increasingly significant when $d / \lambda_{\mathrm{MFP}}>1$. Note that, since higher collisionality shifts the peak of the angular distribution to larger angles [5], the analytic expression $S_{\theta}(\theta)$ becomes, coincidentally, a better approximation to the self-consistent simulation results for a finite collisionality $d / \lambda_{\mathrm{MFP}} \simeq 1$ than the expression $\varrho_{\theta}^{\mathrm{sc}}(\theta)$.

\section{The ratio of scattered to ballistic ions}

As previously noted, the ratios of the density and flux of scattered ions to those of ballistic ions are also of basic interest in various applications [20]. It is clear from the expansion we have employed in Sec. III that the density and flux of scattered ions are of the order of $z / \lambda_{\mathrm{MFP}}$ times those of the ballistic ions. In what follows, we calculate these ratios more precisely using the distribution functions derived in Sec. III.

The number density of ballistic ions at $z=\xi d$ is given in dimensionless form by

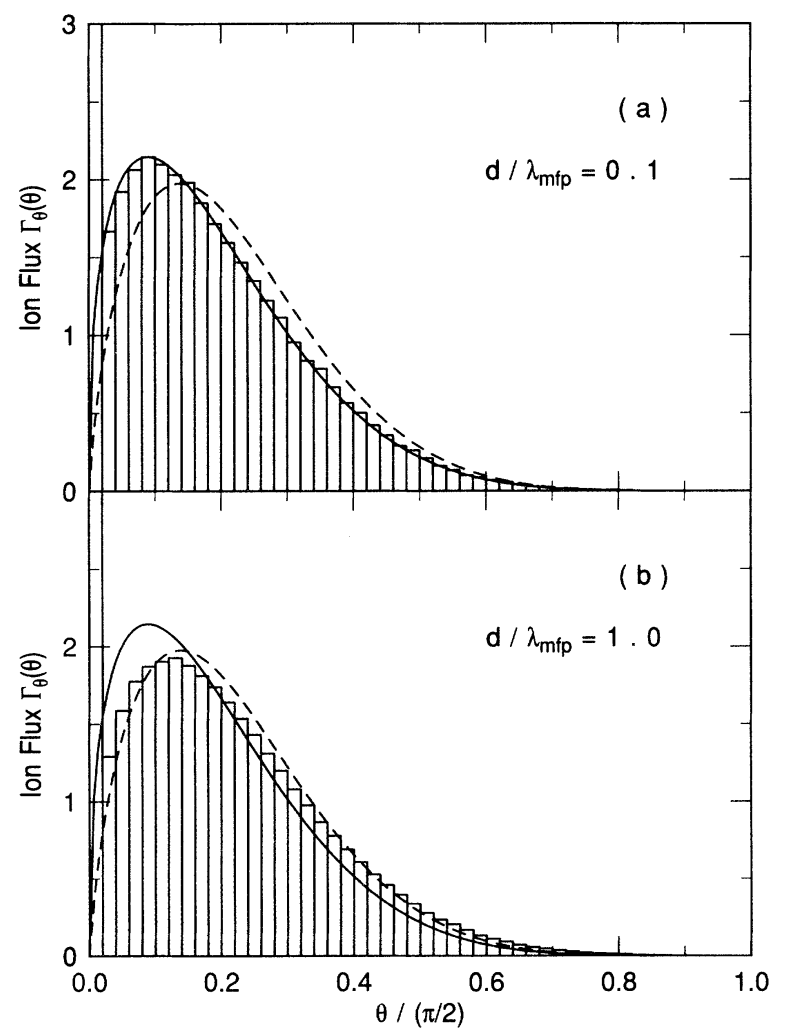

$$
\bar{n}_{\text {ballis }}(\zeta)=\bar{n}_{0}(\zeta)+\left(d / \lambda_{\mathrm{MFP}}\right) \bar{n}_{1}(\zeta),
$$

where

$$
\bar{n}_{0}(\zeta)=\int_{0}^{\infty} \bar{f}_{0} d \mathbf{u}=u_{B} / \sqrt{u_{B}^{2}-2 \phi_{0}(\zeta)}
$$

and

$$
\bar{n}_{1}(\xi)=\int_{0}^{\infty} \bar{f}_{1}^{-} d \mathbf{u}=-\bar{n}_{0}(\xi) \xi
$$

from Eqs. (20) and (34), and thus in dimensional form by

$$
n_{\text {ballis }}(z)=n_{0}(z)\left[1-\frac{z}{\lambda_{\mathrm{MFP}}}\right] \text {. }
$$

We note that $\bar{n}_{1}$ represents the loss of ballistic ions due to collisions. The density of scattered ions is given by

$$
n_{\text {scat }}(z)=\int_{0}^{\pi / 2} F_{\theta}(\theta, z) d \theta .
$$

If the electric field is constant $\left[E(z)=E_{I}\right]$, and the condition (55) is satisfied, then the scattered ion density (75) may be calculated from Eq. (54), and the zeroth-order ion density is given by Eq. (72) as

$$
n_{0}(z)=\frac{n_{I} v_{B}}{\sqrt{2 q E_{I} z / m}} .
$$

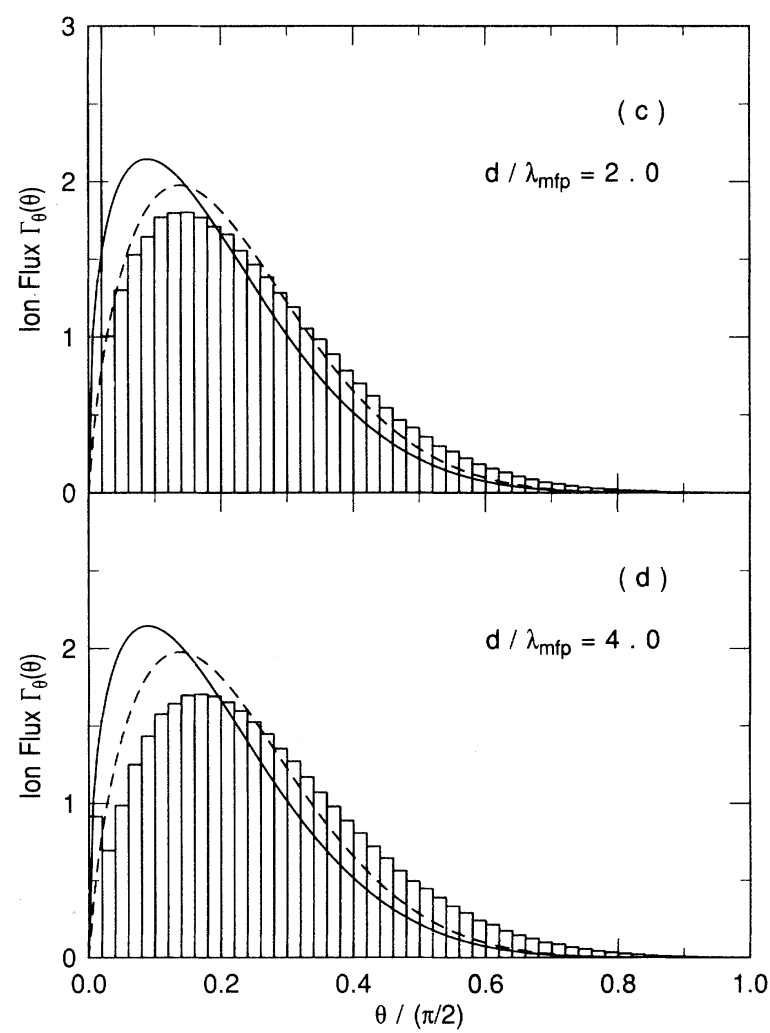

FIG. 5. A comparison of the ion-flux angular distributions at different collisionalities: (a) $d / \lambda_{\mathrm{MFP}}=0.1$; (b) $d / \lambda_{\mathrm{MFP}}=1.0,(\mathrm{c})$ $d / \lambda_{\mathrm{MFP}}=2.0$; and (d) $d / \lambda_{\mathrm{MFP}}=4.0$ for the case of a self-consistent field. The expressions in the low-collisionality limit are also presented [Eq. (68) for the solid lines, and Eq. (57) for the dashed lines]. The ballistic ion component is truncated except for (d) and the magnitude of $\Gamma_{\theta}$ is normalized, as in Figs. 3 and 4 . The other dimensionless parameters used in these calculations are $u_{B}=5.7 \times 10^{-4}, \bar{E}_{I}=2.8 \times 10^{-4}$, and $\zeta=1$. 
Therefore, from Eqs. (54), (74), (75), and (76), we obtain to the lowest order the ratio of the scattered ion density to the ballistic ion density as

$$
\frac{n_{\text {scat }}}{n_{\text {ballis }}}=\frac{z}{\lambda_{\mathrm{MFP}}} \int_{0}^{\pi / 2} \mathcal{F}_{\theta}(\theta) d \theta \simeq 1.39 \frac{z}{\lambda_{\mathrm{MFP}}}
$$

for a constant electric field and a value of $z$ satisfying the condition (55).

Similarly, the ballistic ion flux at $z=\zeta d$ is given in dimensionless form by $\bar{\Gamma}_{\text {ballis }}=\bar{\Gamma}_{0}+\bar{\Gamma}_{1}$, where

$$
\bar{\Gamma}_{0}(\zeta)=\int_{0}^{\infty} u_{z} \bar{f}_{0} d \mathbf{u}=u_{B}
$$

and

$$
\bar{\Gamma}_{1}(\zeta)=\int_{0}^{\infty} u_{z} \bar{f}_{1}^{-} d \mathbf{u}=-u_{B} \zeta,
$$

from Eqs. (20) and (34), and in dimensional form by

$$
\Gamma_{\text {ballis }}(z)=\Gamma_{0}\left(1-\frac{z}{\lambda_{\mathrm{MFP}}}\right) \text { with } \Gamma_{0}=n_{I} v_{B} .
$$

As before, $\bar{\Gamma}_{1}$ represents the loss of ballistic ion flux due to collisions. On the other hand, the $z$ component of the scattered ion flux is given from Eq. (41) by

$$
\Gamma_{\text {scat }}(z)=\int_{0}^{\pi / 2} \Gamma_{\theta}(\theta, z) d \theta=\frac{n_{I} \omega_{p i} d^{2}}{\lambda_{\mathrm{MFP}}} \int u_{z} \bar{f}_{1}^{+} d \mathbf{u} .
$$

However, it is clear from the first-order Boltzmann equation (23) and the initial condition $f_{1}(z=0)=0$ that the first-order flux is zero for every $z$, i.e.,

$$
\int u_{z}\left(\bar{f}_{1}^{+}+\bar{f}_{1}^{-}\right) d \mathbf{u}=0 \Longleftrightarrow \Gamma_{\text {scat }}=-\Gamma_{1},
$$

regardless of whether the electric field is self-consistent. Since expression (34) is the exact solution $\bar{f}_{1}^{-}$of the firstorder equation (32), we obtain

$$
\Gamma_{\text {scat }}=-\Gamma_{1}=\Gamma_{0} \frac{z}{\lambda_{\mathrm{MFP}}}
$$

from Eq. (80) as the exact scattered ion flux. In fact, as a special case, if the electric field is constant $\left[E(z)=E_{I}\right]$ and the condition (55) is satisfied, then we may use the expressions (58) and Eq. (60) to obtain

$$
\Gamma_{\text {scat }}=\Gamma_{0} \frac{z}{\lambda_{\mathrm{MFP}}} \int_{0}^{\pi / 2} \mathcal{G}_{\theta}(\theta) d \theta=\Gamma_{0} \frac{z}{\lambda_{\mathrm{MFP}}} .
$$

From Eqs. (80) and (82), therefore, the ratio of the scattered ion flux to the ballistic ion flux is given to the lowest order by

$$
\frac{\Gamma_{\text {scat }}}{\Gamma_{\text {ballis }}}=\frac{z}{\lambda_{\text {MFP }}}
$$

for any given or self-consistent electric field and at any $z$.

\section{ENERGY DISTRIBUTIONS}

We now calculate the energy distributions of the ion flux incident on the electrodes of the ion density at the electrodes, using the ion distribution function derived in Sec. III. In Sec. V A, a general formulation to derive the energy distributions of the ion flux and the ion density is presented. The cases of a constant electric field and the self-consistent electric field are discussed in Secs. V B and V C, respectively.

\section{A. General formulation}

The energy distributions of the ion density $F_{\text {en }}(\eta, z)$ and of the ion flux $\Gamma_{\text {en }}(\eta, z)$ are defined as

$$
\begin{aligned}
& F_{\mathrm{en}}(\eta, z) d \eta=\int_{0}^{2 \pi} d \varphi \int_{0}^{\pi} \sin \theta d \theta f v^{2} d v, \\
& \Gamma_{\mathrm{en}}(\eta, z) d \eta=\int_{0}^{2 \pi} d \varphi \int_{0}^{\pi} \sin \theta d \theta v_{z} f v^{2} d v,
\end{aligned}
$$

where $\eta$ denotes the ratio of the ion kinetic energy to the kinetic energy of the ballistic ions at $z$, i.e.,

$$
\eta=\frac{\frac{1}{2} m v^{2}}{\frac{1}{2} m v_{B}^{2}-q \Phi_{0}}=\frac{u^{2}}{u_{B}^{2}+y} .
$$

For scattered ions $(\eta<1)$, the normalized ion distribution function is given by Eq. (38) and we have

$$
\begin{aligned}
F_{\mathrm{en}}(\eta, z)= & \frac{d n_{I}\left(u_{B}^{2}+y\right)^{1 / 2}}{\lambda_{\mathrm{MFP}}} \\
& \times \int_{J(\alpha)} \frac{d \theta u_{B} \sin \theta \sqrt{\eta}}{-\phi_{0}^{\prime}\left(\zeta_{c}\right)\left[1-\left(1+\sin ^{2} \theta\right) \eta\right]}, \\
\Gamma_{\mathrm{en}}(\eta, z)= & \frac{d n_{I}\left(u_{B}^{2}+y\right)}{\lambda_{\mathrm{MFP}}} \\
& \times \int_{J(\alpha)} \frac{d \theta u_{B} \sin \theta \cos \theta \eta}{-\phi_{0}^{\prime}\left(\zeta_{c}\right)\left[1-\left(1+\sin ^{2} \theta\right) \eta\right]} .
\end{aligned}
$$

Here we have used the relation

$$
u_{B}^{2}-2\left(u_{\perp}^{2}+\mathscr{E}\right)=\left(u_{B}^{2}+y\right)\left[1-\left(1+\sin ^{2} \theta\right) \eta\right] .
$$

The range of integration $J(\alpha)$ for $\theta$ is obtained from the conditions (45) and (56); combining these conditions yields

$$
\frac{u^{2}}{u_{B}^{2}+y} \leq \cos ^{2} \theta \leq \frac{1}{u_{B}^{2}}\left[u^{2}+\left(u_{B}^{2}+y\right) \frac{y}{u^{2}}-2 y\right]
$$

or

$$
\eta \leq \cos ^{2} \theta \leq \frac{\eta^{2}+(1-2 \eta)\left(1-\alpha^{2}\right)}{\eta \alpha^{2}} .
$$

The condition that the right-hand side of Eq. (88) be larger than 1 or

$$
\eta+\left(\eta^{-1}-2\right)\left(1-\alpha^{2}\right)>\alpha^{2} \Longleftrightarrow(\eta-1)\left(\eta-1+\alpha^{2}\right)>0
$$

is given by $\eta<1-\alpha^{2}$ for $\eta<1$. In other words, the distribution function has the form (38) only for $\theta$ satisfying

$$
\eta \leq \cos ^{2} \theta \leq 1 \text { when } 0 \leq \eta \leq 1-\alpha^{2}
$$

or

$$
\eta \leq \cos ^{2} \theta \leq \frac{\eta^{2}+(1-2 \eta)\left(1-\alpha^{2}\right)}{\eta \alpha^{2}} \text { when } 1-\alpha^{2} \leq \eta<1 .
$$


The range of integration $J(\alpha)$ for $\theta$ must be chosen accordingly.

In the following two subsections, as in the previous section, we shall discuss two different electric-field configurations: a constant electric field and a self-consistent electric field. As in the case of angular distributions, analytic integrations of the expressions for the energy distributions are easily performed for a given constant electric field, and simple analytic formulas for energy distributions are thus obtained. For a self-consistent electric field, integral formulae for $\mathcal{F}_{\text {en }}$ and $\mathcal{S}_{\text {en }}$ will be presented.

\section{B. Constant fields}

In the case of a constant electric field, we may substitute $-\phi_{0}^{\prime}\left(\zeta_{c}\right)=\bar{E}_{I}$ into Eqs. (86) and (87). Using dimensional parameters, we obtain

$$
\begin{aligned}
& F_{\mathrm{en}}(\eta, z)=n_{I} \frac{\frac{1}{2} m v_{B} u_{\mathrm{max}}}{q E_{I} \lambda_{\mathrm{MFP}}} \mathcal{F}_{\mathrm{en}}(\eta ; \alpha), \\
& \Gamma_{\mathrm{en}}(\eta, z)=\Gamma_{0} \frac{\frac{1}{2} m v_{\mathrm{max}}^{2}}{q E_{I} \lambda_{\mathrm{MFP}}} \mathcal{G}_{\mathrm{en}}(\eta ; \alpha),
\end{aligned}
$$

where

$$
\mathcal{F}_{\mathrm{en}}(\eta ; \alpha)=\frac{2}{\sqrt{\eta}} \int_{J(\alpha)} \frac{\sin \theta d \theta}{\cos ^{2} \theta-\left(2-\eta^{-1}\right)},
$$

$$
\mathcal{S}_{\mathrm{en}}(\eta ; \alpha)=2 \int_{J(\alpha)} \frac{\cos \theta \sin \theta d \theta}{\cos ^{2} \theta-\left(2-\eta^{-1}\right)} .
$$

In Eqs. (93) and (94), the denominators of the integrands are expressed in terms of $\cos \theta$, rather than $\sin \theta$, so it is clearly seen that these integrations may be carried out by the substitution of variables $t=\cos \theta$ for Eq. (93) and $t=\cos ^{2} \theta$ for Eq. (94). In Eqs. (91) and (92), $u_{\max }$ denotes the velocity attained by the ballistic ions at $z=\zeta d$, i.e.,

$$
v_{\max }=\sqrt{v_{B}^{2}-2 q \Phi_{0}(z)}=\omega_{p i} d \sqrt{u_{B}^{2}+y} .
$$

In fact, $v_{\max }$ gives the maximum speed attainable by any ion (i.e., including scattered ions) at $z$. For a constant electric field, $E_{I}, v_{\max }$ and $\alpha=v_{B} / v_{\max }$ satisfy a simple relation, namely $v_{\max }^{2}=v_{B}^{2}+2 q E_{I} z / m$ or $E_{I} / v_{\max }^{2}$ $=\left(1-\alpha^{2}\right) m / 2 q z$. Therefore, we may write

$$
\begin{aligned}
& F_{\mathrm{en}}(\eta, z)=\eta_{I} \frac{z}{\lambda_{\mathrm{MFP}}} \frac{\alpha}{\left(1-\alpha^{2}\right)} \mathcal{F}_{\mathrm{en}}(\eta ; \alpha), \\
& \Gamma_{\mathrm{en}}(\eta, z)=\Gamma_{0} \frac{z}{\lambda_{\mathrm{MFP}}} \frac{1}{\left(1-\alpha^{2}\right)} \mathcal{G}_{\mathrm{en}}(\eta ; \alpha) .
\end{aligned}
$$

As noted earlier, $\alpha=v_{B} / v_{\max }$ is a small quantity in most discharge plasmas and the condition $1-\alpha^{2}>\frac{1}{2}$ is generally satisfied. For such $\alpha$, the analytic expression for $\mathcal{F}_{\text {en }}$ after integration is given by

$$
F_{\mathrm{en}}(\eta ; \alpha)=\left\{\begin{array}{l}
\frac{2}{\sqrt{1-2 \eta}}\left[\arctan \left(\frac{\eta}{1-2 \eta}\right)^{1 / 2}-\arctan \frac{\eta}{\sqrt{1-2 \eta}}\right] \quad\left(0 \leq \eta<\frac{1}{2}\right) \\
\frac{1}{\sqrt{2 \eta-1}}\left[\ln \left(\frac{\sqrt{\eta}-\sqrt{2 \eta-1}}{\sqrt{\eta}+\sqrt{2 \eta-1}}\right)-\ln \left(\frac{\eta-\sqrt{2 \eta-1}}{\eta+\sqrt{2 \eta-1}}\right)\right] \quad\left(\frac{1}{2}<\eta \leq 1-\alpha^{2}\right) \\
\frac{1}{\sqrt{2 \eta-1}}\left[\ln \left(\frac{\sqrt{(1-\eta)^{2}-\alpha^{2}(1-2 \eta)}-\alpha \sqrt{\eta(2 \eta-1)}}{\sqrt{(1-\eta)^{2}-\alpha^{2}(1-2 \eta)}+\alpha \sqrt{\eta(2 \eta-1)}}\right]-\ln \left[\frac{\eta-\sqrt{2 \eta-1}}{\eta+\sqrt{2 \eta+1}}\right]\right) \quad\left(1-\alpha^{2}<\eta<1\right) .
\end{array}\right.
$$

If, however, the initial velocity $v_{B}$ is large enough so that $1-\alpha^{2} \leq \frac{1}{2}, \mathcal{F}_{\text {en }}$ has the form

$$
F_{\mathrm{en}}(\eta ; \alpha)=\left\{\begin{array}{l}
\frac{2}{\sqrt{1-2 \eta}}\left[\arctan \left[\frac{\eta}{1-2 \eta}\right]^{1 / 2}-\arctan \frac{\eta}{\sqrt{1-2 \eta}}\right] \quad\left(0 \leq \eta<1-\alpha^{2}\right) \\
\frac{2}{\sqrt{1-2 \eta}}\left[\arctan \left[\frac{\sqrt{(1-\eta)^{2}-\alpha^{2}(1-2 \eta)}}{\alpha \sqrt{1-2 \eta}}\right]-\arctan \left[\frac{\eta}{\sqrt{1-2 \eta}}\right]\right] \quad\left(1-\alpha^{2} \leq \eta<\frac{1}{2}\right) \\
\frac{1}{\sqrt{2 \eta-1}}\left[\ln \left[\frac{\sqrt{(1-\eta)^{2}-\alpha^{2}(1-2 \eta)}-\alpha \sqrt{\eta(2 \eta-1)}}{\sqrt{(1-\eta)^{2}-\alpha^{2}(1-2 \eta)}+\alpha \sqrt{\eta(2 \eta-1)}}\right]-\ln \left[\frac{\eta-\sqrt{2 \eta-1}}{\eta+\sqrt{2 \eta+1}}\right]\right] \quad\left(\frac{1}{2}<\eta<1\right) .
\end{array}\right.
$$

For the energy distribution of the ion flux $\mathcal{S}_{\text {en }}$, we have a much simpler expression after integration:

$$
\mathcal{S}_{\mathrm{en}}(\eta ; \alpha)= \begin{cases}-\ln (1-\eta) & \left(\eta \leq 1-\alpha^{2}\right) \\ -\ln \alpha^{2} & \left(1-\alpha^{2}<\eta<1\right),\end{cases}
$$

which holds for any $\alpha(0<\alpha<1)$. We note that the distri- bution is constant for $1-\alpha^{2}<\eta<1$.

Figure 6 shows energy distributions of the ion density and the ion flux, respectively, in the case of a constant electric field. As in Fig. 3, the solid lines represent the theoretical profiles, $\mathcal{F}_{\mathrm{en}}(\eta)$ for Fig. $6\left(\right.$ a) and $\mathcal{S}_{\mathrm{en}}(\eta)$ for Fig. 6(b), given above, and the histograms are obtained from the Monte Carlo simulations with the same con- 
stant electric field. Good agreement between the theoretically predicted profiles and the Monte Carlo data is seen in these figures, where the simulation parameters are the same as in Fig. 3. In Figs. 6(a) and 6(b), the profiles are normalized such that the areas under the curves and the histograms for scattered ions is unity, and the ballistic component of the Monte Carlo data at $\eta=1$ is truncated by the frame of the figure, as in Fig. 3.

\section{Self-consistent fields}

In the case of a self-consistent electric field, the potential $\phi_{0}$ is obtained by solving Eq. (22). In this case, the electric field $\phi_{0}^{\prime}$ becomes a function of $\eta$ and $\theta$, as discussed in the previous section for angular distributions. The energy distributions $F_{\text {en }}(\eta, z)$ and $\Gamma_{\text {en }}(\eta, z)$ must then be calculated directly from Eqs. (86) and (87) with knowledge of the dependence of $\phi_{0}^{\prime}$ on $\eta$ and $\theta$. As shown in Eqs. $(61)-(62)$, we may write $-\phi_{0}^{\prime}\left(\zeta_{c}\right)$ $=\sqrt{2 \alpha} u_{\max } h(\theta)$, where
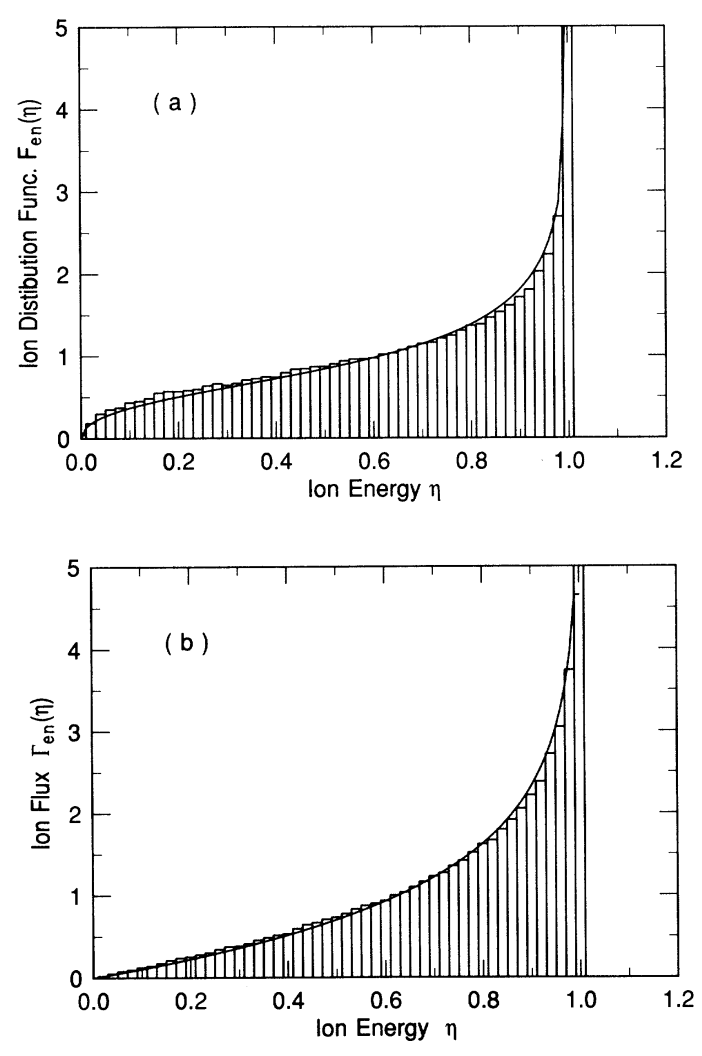

FIG. 6. The energy distribution (a) of the ion density and (b) of the ion flux in the case of a constant electric field obtained from the Monte Carlo simulations (histogram), Eq. (97) [the solid curve in (a)] and Eq. (99) [the solid curve in (b)]. The ballistic ion component, represented by the bin at $\eta=1.0$, is truncated and the magnitudes of $\mathcal{F}_{\text {en }}$ and $\mathcal{S}_{\text {en }}$ are normalized as described in the text. The dimensionless parameters used here are the same as those for Fig. 3. $h(\theta)=\left\{\sqrt{(1-\eta)^{2}\left[1-\left(1+\sin ^{2} \theta\right) \eta\right]}+\alpha K\right\}^{1 / 2}$,

Here the function $h(\theta)$ and the function $g(\xi)$ of Eq. (64) are equivalent, with the relation $\eta=\xi^{2}=u^{2} /\left(u_{B}^{2}+y\right)$. From Eqs. (86) and (87), the energy distribution of the ion density is then given by

$$
F_{\mathrm{en}}(\eta, z)=n_{I} \frac{d}{\lambda_{\mathrm{MFP}}} \frac{u_{B}}{\sqrt{2 \alpha}} \mathcal{F}_{\mathrm{en}}^{\mathrm{sc}}(\eta ; \alpha, K)
$$

and that of the ion flux is given by

$$
\Gamma_{\mathrm{en}}(\eta, z)=\Gamma_{0} \frac{d}{\lambda_{\mathrm{MFP}}} \frac{u_{B}}{\sqrt{2 \alpha^{3}}} \mathcal{Q}_{\mathrm{en}}^{\mathrm{sc}}(\eta ; \alpha, K),
$$

where

$$
\begin{aligned}
& \mathcal{F}_{\mathrm{en}}^{\mathrm{sc}}(\eta ; \alpha, K)=\sqrt{\eta} \int_{J(\alpha)} \frac{\sin \theta d \theta}{h(\theta)\left[1-\left(1+\sin ^{2} \theta\right) \eta\right]}, \\
& \mathcal{G}_{\mathrm{en}}^{\mathrm{sc}}(\eta ; \alpha, K)=\eta \int_{J(\alpha)} \frac{\sin \theta \cos \theta d \theta}{h(\theta)\left[1-\left(1+\sin ^{2} \theta\right) \eta\right]} .
\end{aligned}
$$

Figures $7(\mathrm{a})$ and $7(\mathrm{~b})$ show energy distributions of the
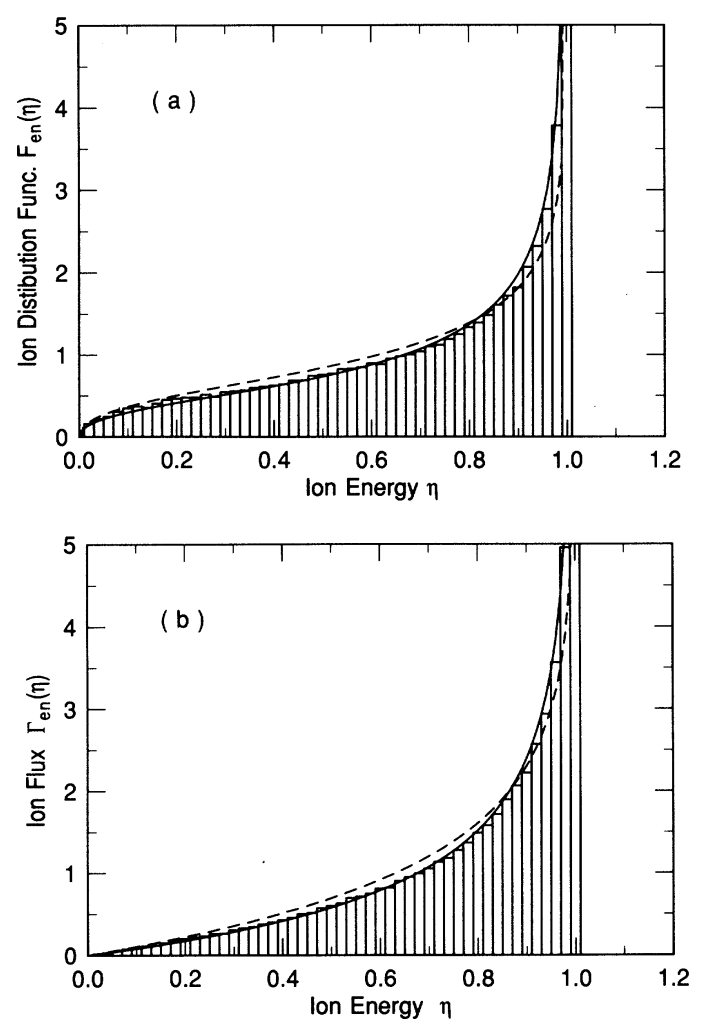

FIG. 7. The energy distributions (a) of the ion density and (b) of the ion flux in the case of a self-consistent electric field obtained from the Monte Carlo simulations (histogram), Eq. (103) [the solid curve in (a)] and Eq. (104) [the solid curve in (b)]. For comparison, the formulas for the constant-field approximation [Eq. (97) for (a) and Eq. (99) for (b)] are also presented as dashed lines. The ballistic ion component, represented by the bin at $\eta=1.0$, is truncated and the magnitudes of $\mathcal{F}_{\text {en }}$ and $\mathcal{S}_{\text {en }}$ are normalized as described in the text. The dimensionless parameters used here are the same as those for Fig. 4. 
ion density and the ion flux, respectively: the histograms are obtained from the Monte Carlo simulations with a self-consistent electric field [5], while the solid curves are calculated from Eqs. (103) and (104). The dashed curves represent constant-field approximations: $\mathcal{F}_{\text {en }}(\eta ; \alpha)$ of Eq. (97) in Fig. 7(a) and $S_{\text {en }}(\eta ; \alpha)$ of Eq. (99) in Fig. 7(b). The distributions are normalized in the usual manner, and the ballistic component is truncated. The parameters used in these calculations are the same as those for Fig. 4. Good agreement between the simulation results and the theoretical expressions ( $\mathscr{F}_{\text {en }}^{\text {sc }}$ and $\mathcal{S}_{\text {en }}^{\text {sc }}$ ) is again evident in Fig. 7, whereas the theoretical models based on the constant-field assumption $\left(\mathscr{F}_{\text {en }}\right.$ and $\left.\mathcal{S}_{\text {en }}\right)$ predict distributions shifted slightly toward lower energies.

Figures 8(a) and 8(b) show a comparison of the analytic expression for the ion-flux energy distribution $\mathcal{G}_{\mathrm{en}}^{\mathrm{sc}}(\eta)$ (solid curves) with the self-consistent Monte Carlo simulation results for various collisionalities: $d / \lambda_{\mathrm{MFP}}=0.1$, 1.0, 2.0, and 4.0. Here, the dashed curves represent the energy distribution $S_{\text {en }}(\eta)$ for the constant-field approximation (99). It is seen from Fig. 8(b) that the analytical expression $g_{\theta}^{\mathrm{sc}}(\theta)$ fails to approximate the ion-flux energy distribution well at collisionalities $d / \lambda_{\mathrm{MFP}} \simeq 1$ or larger.

\section{DISCUSSION AND CONCLUSIONS}

In this paper we have derived, in the limit of low collisionality, an analytic expression for the ion distribution function in a steady-state sheath from the appropriate kinetic equations with elastic collisions. We have also calculated the angular and energy distributions of the ion flux and the ion density from the analytic form for the ion distribution function. The angular and energy distributions of the ion flux incident on the electrodes are of practical interest in the plasma processing of semiconductor materials.

To summarize the ion-flux distributions in the case of a constant electric field, we have the angular distribution $\Gamma_{\theta}(\theta, z)$ and energy distribution $\Gamma_{\mathrm{en}}(\eta, z)$ at the position $z=\zeta d$ for $\alpha<1$ :

$$
\begin{aligned}
& \Gamma_{\theta}(\theta, z)=\Gamma_{0} \frac{z}{\lambda_{\mathrm{MFP}}} \varrho_{\theta}(\theta), \\
& \Gamma_{\mathrm{en}}(\eta, z)=\Gamma_{0} \frac{z}{\lambda_{\mathrm{MFP}}} \Theta_{\mathrm{en}}(\eta ; \alpha),
\end{aligned}
$$

where
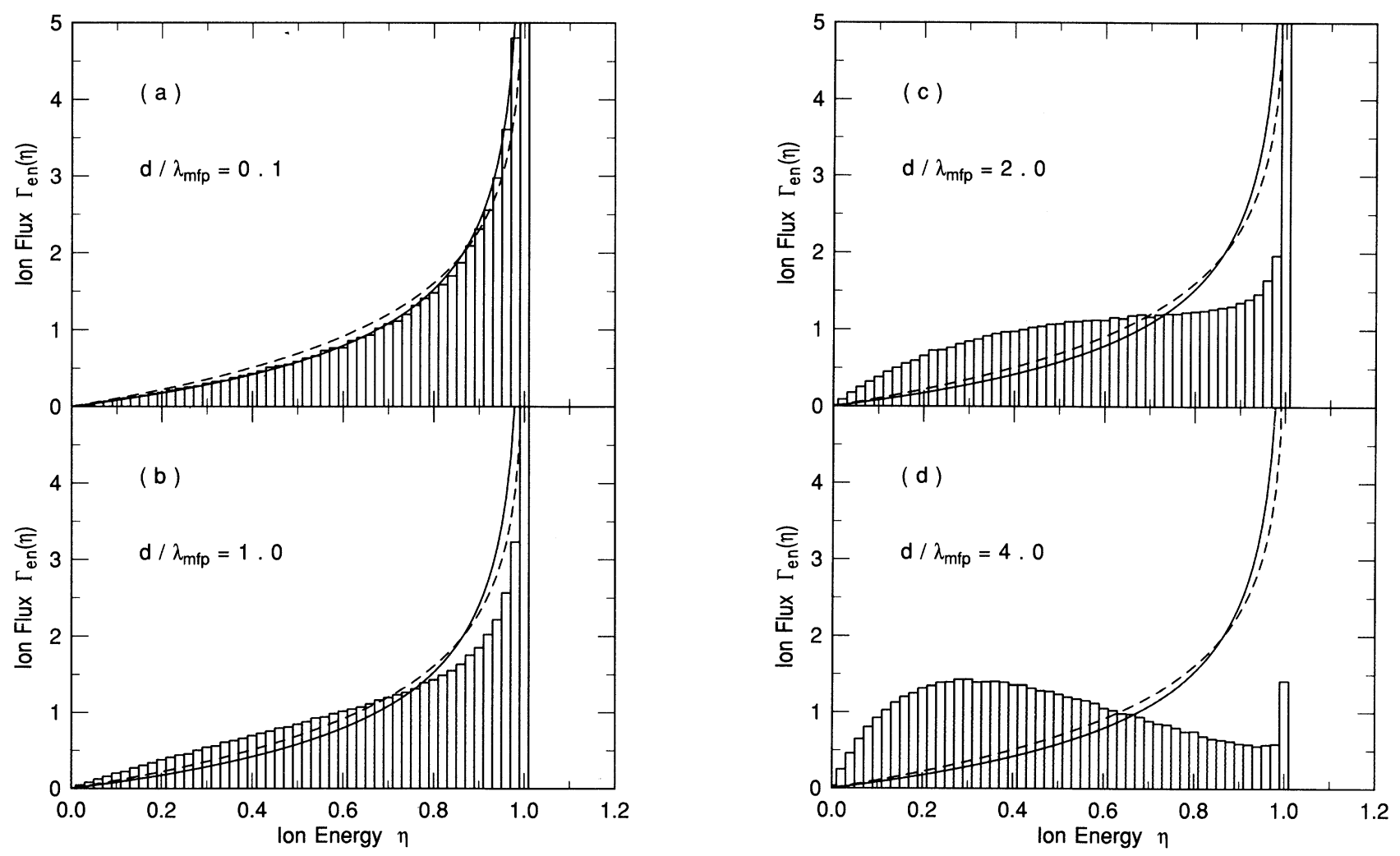

FIG. 8. A comparison of the ion-flux energy distributions at different collisionalities: (a) $d / \lambda_{\mathrm{MFP}}=0.1 ;(\mathrm{b}) d / \lambda_{\mathrm{MFP}}=1.0 ;(\mathrm{c})$ $d / \lambda_{\mathrm{MFP}}=2.0$; and (d) $d / \lambda_{\mathrm{MFP}}=4.0$ in the case of a self-consistent field. The expressions in the low-collisionality limit are also presented [Eq. (104) for the solid lines and Eq. (99) for the dashed lines]. The ballistic ion component is truncated except in (d) and the magnitude of $\mathcal{S}_{\mathrm{en}}$ is normalized, as in Figs. 6 and 7. The other dimensionless parameters used in these calculations are the same as those for Fig. 5. 


$$
\begin{aligned}
& \mathcal{S}_{\theta}(\theta)=\frac{2 \sin \theta \cos \theta}{\left(1+\sin ^{2} \theta\right)^{2}}\left[4 \ln \frac{1}{\sin \theta}+\sin ^{4} \theta-1\right], \\
& \mathcal{S}_{\mathrm{en}}(\eta ; \alpha)= \begin{cases}-\ln (1-\eta) & \left(\eta \leq 1-\alpha^{2}\right) \\
-\ln \alpha^{2} & \left(1-\alpha^{2}<\eta<1\right),\end{cases}
\end{aligned}
$$

from Eqs. (57), (58), (96), and (99).

In the case of the self-consistent field, we have obtained the following integral forms for the ion-flux distributions [see Eqs. (64), (66), (68), (100), (102), and (104)]:

$$
\Gamma_{\theta}(\theta, z)=\Gamma_{0} \frac{d}{\lambda_{\mathrm{MFP}}} \frac{u_{B}}{\sqrt{2 \alpha^{3}}} \mathcal{S}_{\theta}^{\mathrm{sc}}(\theta ; \alpha, K),
$$

where

$$
\begin{aligned}
& \mathcal{S}_{\theta}^{\mathrm{sc}}(\theta ; \alpha, K)=2 \sin \theta \cos \theta \int_{I(\alpha)} \frac{\xi^{3} d \xi}{g(\xi)\left[1-\left(1+\sin ^{2} \theta\right) \xi^{2}\right]}, \\
& g(\xi)=\left\{\sqrt{\left(1-\xi^{2}\right)^{2} /\left[1-\left(1+\sin ^{2} \theta\right) \xi^{2}\right]}+\alpha K\right\}^{1 / 2},
\end{aligned}
$$

and

$$
\Gamma_{\mathrm{en}}(\eta, z)=\Gamma_{0} \frac{d}{\lambda_{\mathrm{MFP}}} \frac{u_{B}}{\sqrt{2 \alpha^{3}}} \mathcal{G}_{\mathrm{en}}^{\mathrm{sc}}(\eta ; \alpha, K),
$$

where

$$
\begin{aligned}
& \mathcal{G}_{\mathrm{en}}^{\mathrm{sc}}(\eta ; \alpha, K)=\eta \int_{J(\alpha)} \frac{\sin \theta \cos \theta d \theta}{h(\theta)\left[1-\left(1+\sin ^{2} \theta\right) \eta\right]}, \\
& h(\theta)=\left\{\sqrt{(1-\eta)^{2} /\left[1-\left(1+\sin ^{2} \theta\right) \eta\right]}+\alpha K\right\}^{1 / 2} .
\end{aligned}
$$

Here the ranges of integration $I(\alpha)$ and $J(\alpha)$ are given by Eqs. (69) and (70), and by Eqs. (89) and (90), respectively.

The analytic expressions for the ion flux given above offer an inexpensive means of describing the angular and energy distributions of ions bombarding the electrodes, in the limit of weak collisionality, and may be used as input to shape-evolution calculations such as those described in [20]. We have also derived analytic expressions for the distributions of the ion density, given by Eqs. (52), (53), (95), (97), and (98) for constant electric fields, and by Eqs. (64), (67), (101), and (103) for self-consistent fields.

It is interesting to observe the shape of the angular and energy distributions of the ion flux for non-negligible values of $\alpha$. Figure 9 shows results for a self-consistent electric field with the value $\alpha=0.32$. The parameters used in these calculations are: $d / \lambda_{\mathrm{MFP}}=0.14, u_{B}=0.34$, $\bar{E}_{I}=0.17, \zeta=d / \lambda_{\mathrm{MFP}}=1, K=-0.75$, and $-\phi_{0}=0.50$. In dimensional quantities, these calculations correspond to an Ar discharge $\left(\sigma_{\text {tot }}=5.0 \times 10^{-15} \mathrm{~cm}^{2}\right)$ with gas pressure $p_{g}=10 \mathrm{mTorr}$, neutral gas number density $n_{g}=2.7$ $\times 10^{14} \mathrm{~cm}^{-3}$, ionization rate $n_{i} / n_{g}=9.0 \times 10^{-6}$, sheath thickness $d=1 \mathrm{~mm}$, mean free path $\lambda_{\mathrm{MFP}}=7.3 \mathrm{~mm}$, electron temperature $T_{e}=5 \mathrm{eV}$, and sheath potential drop $-\Phi=22 \mathrm{~V}$.

The nonsmooth behavior observed at $\theta=\theta_{c}=0.16[\mathrm{Eq}$. (71)] and at $\eta=1-\alpha^{2}=0.90$ in these figures is an artifact of the assumption of a sharp presheath-sheath boundary at $z=0$; in our model, the ions are assumed to have a given distribution function [i.e., the $\delta$ function (20)] for $z \leq 0$ and suffer from collisions with neutrals only after entering the sheath, i.e., for $z>0$. Physically, in the presence of electrons and neutrals in the presheath and presheath-sheath boundary regions, the nonsmooth connections of the distributions at $\theta=\theta_{c}$ and $\eta=1-\alpha^{2}$ are expected to be somewhat smoothed out. However, a sharp drop in the electron density at the presheath-sheath boundary will nevertheless probably cause an effect somewhat similar to that observed in Fig. 9, i.e., different characteristics for the angular distribution between $\theta \leq \theta_{c}$ and $\theta \geq \theta_{c}$, and of the energy distribution between $\eta \leq 1-\alpha^{2}$ and $\eta \geq 1-\alpha^{2}$.

Incorporating a phenomenological description of electrons in our models (e.g., a Boltzmann distribution) is a relatively simple manner, requiring only a small change in the Poisson equation (3), and would not alter the form of the ion distribution function given in Eq. (38). In the interest of clarity and simplicity, however, we have chosen to neglect the electrons in this study. More realistic presheath-sheath boundary conditions, together with the effects of a finite electron density and other electronrelated phenomena, are planned to be considered in future studies.
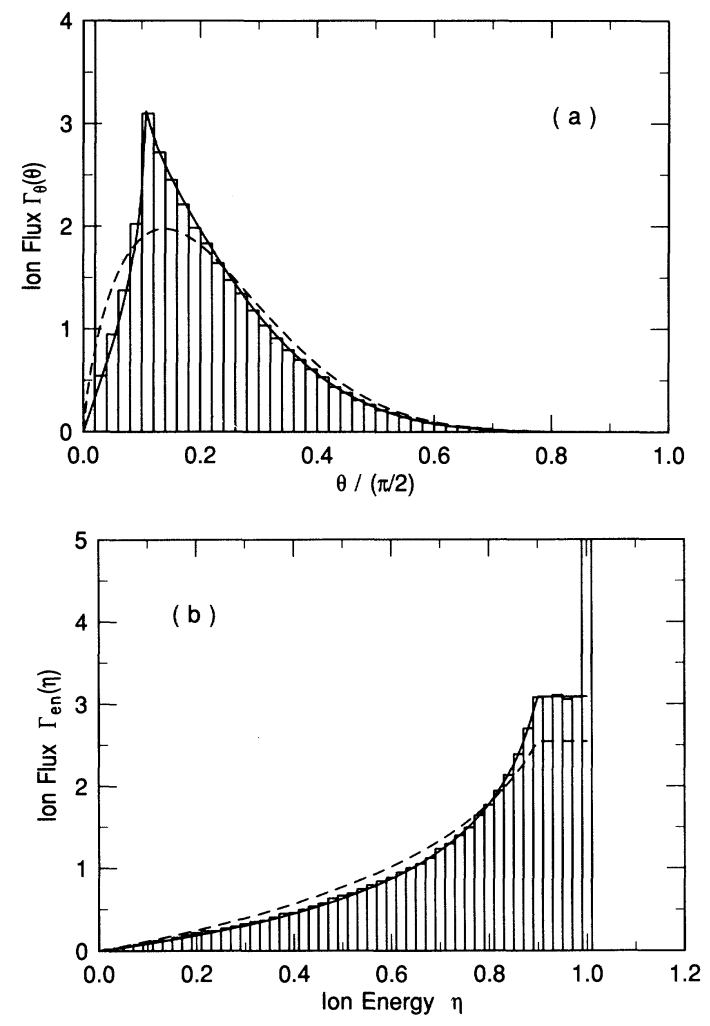

FIG. 9. The angular and energy distributions of the ion flux in the case of a self-consistent electric field (histograms and solid curves) with a finite $\alpha=0.32$ for $\alpha$. The parameters used in these calculations are: $d / \lambda_{\text {MFP }}=0.14, u_{B}=0.34, \bar{E}_{I}=0.17$, $\zeta=d / \lambda_{\mathrm{MFP}}=1, K=-0.75$, and $-\phi_{0}=0.50$. Nonsmooth connections of the distributions curves are observed at $\theta=\theta_{c}=0.16$ and at $\eta=1-\alpha^{2}=0.90$. For comparison, the formulas for the constant-field approximation [Eq. (57) for (a) and Eq. (99) for (b)] are also presented as dashed lines. 


\section{APPENDIX: AN ALTERNATIVE DERIVATION OF THE ION DISTRIBUTION FUNCTION}

In Sec. III, the ion distribution function was derived directly from the Boltzmann equation, making use of the small parameter $d / \lambda_{\mathrm{MFP}}$. Although the mathematical procedure presented there is straightforward, not much attention was paid to clarifying the physical meaning of the derived distribution function. In this appendix we shall employ a more heuristic method to rederive the ion distribution function that gives a clearer physical picture of colliding ions in a weakly collisional sheath. For simplicity, we consider only the distribution function $f^{*}$ of ions that have suffered a single collision with the neutrals.

The number of ballistic ions that collide with neutrals per unit time between $z_{c}$ and $z_{c}+d z_{c}$ and are scattered by a polar angle between $\theta_{c}$ and $\theta_{c}+d \theta_{c}$ is given from Eqs. (9) and (10) by

$$
d N=\Gamma_{0} n_{g} \sigma_{\text {tot }} \sin 2 \theta_{c} d \theta_{c} d z_{c},
$$

if the ions and neutrals have the same mass $(m=M)$. As previously noted, the ion flux is conserved: $\Gamma_{0}$ $=n_{I} v_{B}=n_{0}(z) v_{0}(z)$ is independent of $z$. Since the normalized speed of the ballistic ions travelling in the $z$ direction at $\zeta_{c}=z_{c} / d$ is given by $u_{0}=\sqrt{u_{B}^{2}-2 \phi_{0}\left(\zeta_{c}\right)}$, the ion velocity just after the collision is given from Eq. (10) by

$$
u_{\perp}=\sqrt{u_{B}^{2}-2 \phi_{0}\left(\zeta_{c}\right)} \cos \theta_{c} \sin \theta_{c}
$$

and

$$
u_{z}=\sqrt{u_{B}^{2}-2 \phi_{0}\left(\zeta_{c}\right)} \cos ^{2} \theta_{c} .
$$

These ions are then accelerated in the $z$ direction by the electric field and attain the following normalized velocity $\left(u_{\perp}, u_{z}\right)$ and speed $u=\sqrt{u_{\perp}^{2}+u_{z}^{2}}$ at $\zeta\left(\geq \zeta_{c}\right)$ :

$$
\begin{aligned}
& u_{\perp}^{2}=\mathcal{A} \cos ^{2} \theta_{c} \sin ^{2} \theta_{c}, \\
& u_{z}^{2}=\mathcal{A} \cos ^{4} \theta_{c}+\mathscr{B}, \\
& u^{2}=\mathcal{A} \cos ^{2} \theta_{c}+\mathscr{B},
\end{aligned}
$$

where $\mathcal{A}=u_{B}^{2}-2 \phi_{0}\left(\zeta_{c}\right)$ and $\mathcal{B}=-2\left[\phi_{0}(\xi)-\phi_{0}\left(\zeta_{c}\right)\right]$. On the other hand, $d N$ is related to the distribution function through

$$
d N=u_{z} f^{*} 2 \pi v_{\perp} d v_{\perp} d v_{z} .
$$

Comparing Eq. (A1) with Eq. (A5), we obtain an expression for the distribution function:

$f^{*}\left(u_{\perp}, u_{z}\right)=\frac{\Gamma_{0}}{\left(\omega_{p i} d\right)^{4}} \frac{d}{\lambda_{\mathrm{MFP}}} \frac{\sin 2 \theta_{c}}{2 \pi u_{\perp} u_{z}\left|\partial\left(u_{\perp}, u_{z}\right) / \partial\left(\theta_{c}, \zeta_{c}\right)\right|}$.

From Eqs. (A2) and (A3), the Jacobian in Eq. (A6) is found to be

$$
\left|\frac{\partial\left(u_{\perp}, u_{z}\right)}{\partial\left(\theta_{c}, \zeta_{c}\right)}\right|=\frac{u_{\perp} \sin ^{3} \theta_{c}}{u_{z} \cos \theta_{c}}\left[-\phi^{\prime}\left(\zeta_{c}\right)\right] .
$$

Using the relation
$u_{B}^{2}-2\left(\mathscr{E}+u_{\perp}^{2}\right)=u_{B}^{2}-\left[u_{z}^{2}+2 u_{\perp}^{2}-2 \phi_{0}(\zeta)\right]=u_{\perp}^{2} \frac{\sin ^{2} \theta_{c}}{\cos ^{2} \theta_{c}}$,

we obtain from Eqs. (A6) and (A7)

$f^{*} \frac{\left(\omega_{p i} d\right)^{3}}{n_{I}}=\frac{d}{\lambda_{\mathrm{MFP}}} \frac{u_{B}}{\left[-\pi \phi_{0}^{\prime}\left(\zeta_{c}\right)\right]\left[u_{B}^{2}-2\left(\mathscr{E}+u_{\perp}^{2}\right)\right]}$,

which is equivalent to $\left(d / \lambda_{\mathrm{MFP}}\right) \bar{f}_{1}^{+}$[see Eq. (38)].

The derivation of the distribution function presented above, however, does not clarify possible values of the ion velocity $\left(u_{1}, u_{z}\right)$ [or equivalently $\left(u_{1}, \mathscr{E}\right)$ ] that the ions may attain at $\zeta$. In fact, as we have shown in Sec. III, the distribution function $\bar{f}_{1}^{+}$is given by Eq. (38) only inside the hatched region of Fig. 2 (which corresponds to possible values of ion velocities at $\zeta$ ) and $\bar{f}_{1}^{+}$takes the value 0 outside this region. Starting from Eqs. (A2)-(A4), therefore, we now examine the region in which Eq. (112) is valid and rederive the hatched region of Fig. 2. In particular, we are interested in demonstrating by following an ion motion given by Eqs. (A2)-(A4) that the range of $u$ given by the inequalities (47) and (48) (or equivalently the hatched region in Fig. 2) corresponds to possible values of the speed of an ion that has undergone a single collision.

Writing $u_{\perp}=u \sin \theta$ and $u_{z}=u \cos \theta$, where $\theta$ denotes the velocity angle at $\zeta$, and eliminating $\theta_{c}$ from Eqs. (A2) and (A4), we obtain the following quadratic equation for $u^{2}$ :

$$
u^{4}-\left(\mathcal{A} \cos ^{2} \theta+2 \mathcal{B}\right) u^{2}+(\mathcal{A}+\mathscr{B}) \mathcal{B}=0,
$$

with $\mathcal{C}=\mathcal{A}+\mathcal{B}=u_{B}^{2}-2 \phi_{0}(\xi)$ being independent of $\xi_{c}$. The two solutions of this quadratic equation are given by

$$
u^{2}=\frac{1}{2} \mathcal{A} \cos ^{2} \theta \pm \frac{1}{2} \sqrt{D_{u}}+\mathscr{B}
$$

where the discriminant is given by

$$
\begin{aligned}
D_{u} & =\mathcal{A}^{2} \cos ^{4} \theta-4 \mathcal{A} \mathcal{B} \sin ^{2} \theta \\
& =\mathcal{A}\left[\mathcal{A}\left(1+\sin ^{2} \theta\right)^{2}-4 \mathcal{C} \sin ^{2} \theta\right] .
\end{aligned}
$$

Since $\mathcal{A}, \mathscr{B}>0$, it is easy to see that $0 \leq \cos ^{2} \theta_{c}$ $=\left(u^{2}-\mathscr{B}\right) / \mathcal{A} \leq 1$, so that if $D_{u}>0$, the two solutions given in Eq. (A10) are in fact physically meaningful and there are two different scattering angles $\theta_{c}$ for a given combination of the collision position $\zeta_{c}$ and the final angle $\theta$ at $\zeta$. By varying $\zeta_{c}$ from 0 to $\zeta$ or, equivalently, $\mathcal{A}$ from $u_{B}^{2}$ to $\mathcal{C}$, we can determine the range of variation of $u$. Since $D_{u}$ is a monotonically increasing function of $\mathcal{A}$ for $u_{B}^{2} \leq \mathcal{A} \leq \mathcal{C}$, the minimum value of $D_{u}$ is given by

$$
D_{\min }=u_{B}^{2}\left[u_{B}^{2}\left(1+\sin ^{2} \theta\right)^{2}-4 \odot \sin ^{2} \theta\right]
$$

at $\mathcal{A}=u_{B}^{2}$. Thus we obtain

if $\frac{u_{B}^{2}}{4 y}<\frac{\sin ^{2} \theta}{\cos ^{4} \theta}\left(\Longrightarrow D_{u}<0\right)$ then $0 \leq u^{2} \leq\left(u_{B}^{2}+y\right) \cos ^{2} \theta$

and 
if $\frac{u_{B}^{2}}{4 y} \geq \frac{\sin ^{2} \theta}{\cos ^{4} \theta}\left(\Longleftrightarrow D_{u} \geq 0\right)$

then

$$
\begin{aligned}
& 0 \leq u^{2} \leq y+\frac{u_{B}^{2}}{2} \cos ^{2} \theta-\frac{1}{2} \sqrt{D}, \\
& y+\frac{u_{B}^{2}}{2} \cos ^{2} \theta+\frac{1}{2} \sqrt{D} \leq u^{2} \leq\left(u_{B}^{2}+y\right) \cos ^{2} \theta,
\end{aligned}
$$

which are equivalent to the inequalities (47) and (48).

We have thus shown that the distribution function of the ions that undergo a single collision is obtainable by following the motion of a single ion together with the scattering probability given by Eq. (A1) and, in fact, this matches the ion distribution function obtained from the Boltzmann equation under the assumption of weak collisionality.
[1] W. M. Davis and T. A. Vanderslice, Phys. Rev. 131, 219 (1963).

[2] M. J. Kushner, J. Appl. Phys. 58, 4024 (1985).

[3] B. E. Thompson, H. H. Sawin, and D. A. Fisher, J. Appl. Phys. 63, 2241 (1988).

[4] J. Liu, G. L. Huppert, and H. H. Sawin, J. Appl. Phys. 68, 3916 (1990).

[5] R. T. Farouki, S. Hamaguchi, and M. Dalvie, Phys. Rev. A (to be published).

[6] M. J. Druyvesteyn and F. M. Penning, Rev. Mod. Phys. 12, 87 (1940).

[7] H. Margenau, Phys. Rev. 73, 297 (1948).

[8] H. Margenau and L. M. Hartman, Phys. Rev. 73, 309 (1948).

[9] P. L. Bhatnagar, E. P. Gross, and M. Krook, Phys. Rev. 94, 511 (1954).

[10] H. Dreicer, Phys. Rev. 117, 343 (1960).

[11] G. H. Wannier, Bell Syst. Tech. J. 32, 170 (1953).

[12] J. E. Lawler, Phys. Rev. A 32, 2977 (1985).

[13] H. U. Mittmann and H. P. Weise, Z. Naturforsch. 29, 400 (1974).
[14] M. L. Vestal, C. R. Blakley, and J. H. Futrell, Phys. Rev. A 17, 1337 (1978).

[15] T. J. Moratz, L. C. Pitchford, and J. N. Bardsley, J. Appl. Phys. 61, 2146 (1987).

[16] T. J. Sommerer, W. N. Hitchon, and J. E. Lawler, Phys. Rev. A 39, 6356 (1989)

[17] J. V. DiCarlo and M. Kushner, J. Appl. Phys. 66, 5763 (1989).

[18] J. W. Coburn and E, Kay, J. Appl. Phys. 43, 4965 (1972).

[19] W. M. Holber and J. Forster, J. Vac. Sci. Technol. A 8, 3720 (1990).

[20] M. Dalvie, R. T. Farouki, and S. Hamaguchi (unpublished).

[21] W. P. Allis, in Handbuch der Physik (Springer-Verlag, Berlin, 1956), Vol. 21, p. 383.

[22] See, for example, L. D. Landau and E. M. Lifshitz, Mechanics (Pergamon, Oxford, 1960).

[23] R. T. Farouki, M. Dalvie, and L. F. Pavarino, J. Appl. Phys. 68, 6106 (1990).

[24] B. Chapman, Glow Discharge Processes (Wiley, New York, 1980). 